\title{
A novel tepovirus, Agave virus T, identified by the analysis of the transcriptome data of blue agave (Agave tequilana)
}

\author{
Chul Jun Goh, Dongbin Park, Yoonsoo Hahn* \\ Department of Life Science, Chung-Ang University, Seoul 06974, Republic of Korea
}

Received January 8, 2021; accepted January 19, 2021

\begin{abstract}
Summary. - The genome sequence of a novel RNA virus was identified by analyzing transcriptome data obtained from the stem sample of a blue agave (Agave tequilana) plant. Sequence comparison and phylogenetic analysis showed that the RNA virus, Agave virus T (AgVT), was a new member of the genus Tepovirus in the family Betaflexiviridae. AgVT genome had three open reading frames: a 1605-amino acid (aa) replicase (REP), 355-aa movement protein (MP), and 220-aa coat protein (CP). Phylogenetic analyses based on the REP, MP, and CP sequences of AgVT, previously reported tepoviruses, and other Betaflexiviridae viruses revealed that tepoviruses could be classified into two subclades: "potato virus T (PVT)-clade" and "Prunus virus T (PrVT)-clade." PVT, the type species and founding member of the genus Tepovirus, belong to "PVT-clade" along with AgVT, while the other five tepoviruses belong to "PrVT-clade." The genome sequence of AgVT may be useful for studying the phylogenetic relationships between tepoviruses and other closely related viruses.
\end{abstract}

Keywords: Agave virus T; Tepovirus; Betaflexiviridae; blue agave; Agave tequilana

\section{Introduction}

Tepoviruses (the genus Tepovirus) are plant-infecting RNA viruses of the family Betaflexiviridae. The members of this family have a monopartite, positive-sense, singlestranded RNA genome of size 6-9 kb, and the virions exist as flexuous filamentous particles (Adams et al., 2012; Rubino et al., 2012). Thirteen genera in the family Betaflexiviridae have been reported and were classified into two subfamilies: Trivirinae and Quinvirinae (https:// talk.ictvonline.org, last accessed on December 5, 2020).

The subfamily Trivirinae comprises 10 genera, including Capillovirus, Chordovirus, Citrivirus, Divavirus,

"Corresponding author. E-mail: hahny@cau.ac.kr; phone: +822-820-5812.

Abbreviations: $\mathrm{AgVT}=$ Agave virus $\mathrm{T} ; \mathrm{CP}=$ coat protein; $\mathrm{GVA}=$ grapevine virus $\mathrm{A} ; \mathrm{MP}=$ movement protein $; \mathrm{ORF}=$ open reading frame; $\mathrm{PrVT}=$ Prunus virus T; $\mathrm{PVT}=$ potato virus $\mathrm{T} ; \mathrm{RdRp}=$ RNA-dependent RNA polymerase; REP = replicase
Prunevirus, Ravavirus, Tepovirus, Trichovirus, Vitivirus, and Wamavirus. Viruses of the subfamily Trivirinae have three common open reading frames (ORFs) that encode a replicase (REP), movement protein (MP), and coat protein (CP) (Adams et al., 2012; Rubino et al., 2012). Members of the genera Capillovirus and Divavirus have a fused REP-CP ORF, while those of the other genera have independent REP and CP ORFs (Yoshikawa et al., 1992; Goh et al., 2018). The subfamily Quinvirinae consists of three genera: Carlavirus, Foveavirus, and Robigovirus. Viruses of the subfamily Quinvirinae have five common ORFs encoding an REP, three triple gene block proteins (TGB1, TGB2, and TGB3), and a CP (Morozov and Solovyev, 2003; Prosser et al., 2015). Members of some genera have one or more additional ORFs (Martelli et al., 1997; Adams et al., 2012; Veerakone et al., 2018).

Blue agave (Agave tequilana), also known as tequila agave, belongs to the genus Agave, which are succulent monocotyledonous plants native to the arid regions of North America (Coleman-Derr et al., 2016). Agave species are highly tolerant to drought and heat stress, because 
they employ the crassulacean acid metabolism in photosynthesis, which enhances water-use efficiency (Borland et al., 2009). Blue agave is an economically important plant in Mexico used in the production of the popular distilled spirit tequila (Cedeno, 1995). Because of its economic importance, blue agave has been subjected to various molecular genetic studies, including transcriptome analyses (Gross et al., 2013; Coleman-Derr et al., 2016; Huang et al., 2018).

Transcriptome data obtained from samples of plant tissue infected with RNA viruses often contain virusderived reads (Nibert et al., 2016). Contig assembly of plant transcriptome data and comprehensive sequence analysis have yielded numerous complete genome sequences of novel RNA viruses (Kim et al., 2018; Park et al., 2018, 2020; Goh et al., 2019, 2020). In this study, we identified the genome sequence of a novel virus belonging to the genus Tepovirus of the family Betaflexiviridae in the transcriptome dataset acquired from the stem sample of a blue agave plant (Gross et al., 2013).

\section{Materials and Methods}

The transcriptome data (a total of 71.8 gigabases) obtained using the samples from the stem of a blue agave plant (Gross et al., 2013) were downloaded from the Sequence Read Archive (SRA) of the National Center for Biotechnology Information (NCBI). SRA Acc. Nos. are SRR789714, SRR789715, SRR789716, SRR789717, SRR789718, SRR789719, SRR789720, SRR789724, SRR789725, SRR789726, SRR789727, and SRR789728. Low-quality reads were filtered out using the sickle program (version 1.33; https:/github.com/najoshi/sickle) with the parameter “-q 30 -1 55." High-quality reads from all 12 sequencing runs were pooled and subjected to de novo transcriptome assembly using the SPAdes Genome Assembler (version 3.14.1; http://cab.spbu.ru/ software/spades) with the parameter "--rna."

The transcriptome contigs were compared with known viral RNA-dependent RNA polymerase (RdRp) domain sequences using the DIAMOND program (version 2.0.4; http://www.diamondsearch.org/index.php). A total of 2565 viral RdRp domain sequences were selected from 22 families (PF00602, PF00603, PF00604, PF00680, PF00946, PF00972, PF00978, PF00998, PF02123, PF03035, PF03431, PF04196, PF04197, PF05788, PF05919, PF06317, PF07925, PF08467, PF08716, PF08717, PF12426, and PF17501) available in the Pfam database (release 33.1; https:// pfam.xfam.org).

Sequencing depth of a putative viral contig was assessed by mapping the RNA-seq reads to the contig sequence using the URMAP program (version 1.0.1480; https://drive5.com/urmap). Putative ORFs in the viral genome sequence were inferred based on BLASTX search results against all known viral protein sequences. Conserved domains in protein sequences were predicted using the InterPro web server (version 82.0; https:// www.ebi.ac.uk/interpro).

Pairwise identities of protein sequences were examined using the needle program of the EMBOSS package (version 6.6.0.0; http://emboss.open-bio.org) using default parameters. Multiple sequence alignments were generated using the MAFFT program (version 7.475; https://mafft.cbrc.jp/alignment/software) with the parameter "--auto." Phylogenetic analysis was performed using the neighbor-joining method in the ClustalW2 program (version 2.1; http://www.clustal.org/clustal2), after removing gaps in the sequence alignment.

\section{Results and Discussion}

RNA-seq reads obtained from the blue agave stem sample were assembled into contigs (Gross et al., 2013). When these contig sequences were compared with known viral RdRp sequences, numerous contigs were identified to contain an RdRp domain. Among them, a 6408-bp contig showed strong sequence similarity to that of the RdRp domain of the potato virus T (PVT) REP sequence (UniProt Acc. No. B5ACE2) (Russo et al., 2009). PVT is the type species of the genus Tepovirus of the family Betaflexiviridae, suggesting that the contig was derived from a virus related to the genus Tepovirus.

A BLASTX search of the NCBI protein database using the 6408-bp contig as a query confirmed that it had ORFs with the highest sequence similarities to proteins encoded by the PVT genome sequence. Therefore, this contig was considered the genome sequence of a novel virus belonging to the genus Tepovirus of the family Betaflexiviridae, and it was tentatively named as Agave virus $\mathrm{T}$ (AgVT). The genome sequence of this virus was deposited in the NCBI database (Acc. No. MW323519).

AgVT genome was predicted to have three complete protein-coding ORFs (Fig. 1 and Table 1): ORF1, encoding a 1605-amino acid (aa) REP; ORF2, a 355-aa MP; and ORF3, a 220-aa CP. The MP ORF overlapped with the REP and CP ORFs. AgVT proteins were predicted to possess conserved domains that are typically present in other members of the family Betaflexiviridae. REP had a viral methyltransferase domain (InterPro Acc. No. IPR002588) at aa positions 43-329, a "viral RNA helicase core domain" (IPR027351) at aa positions 761-1077, and an "viral RNAdependent RNA polymerase domain" (IPR001788) at aa positions 1252-1493. MP had a "viral movement protein domain" (IPR028919) at aa positions 11-194. CP had a "viral coat protein domain" (IPR008879) at aa positions 40-215.

For sequence comparison and phylogenetic analysis, genome and protein sequences of previously reported tepoviruses, including four PVT, two Prunus virus $\mathrm{T}$ (PrVT), two Zostera virus $\mathrm{T}$ (ZoVT), one cherry virus $\mathrm{T}$ 


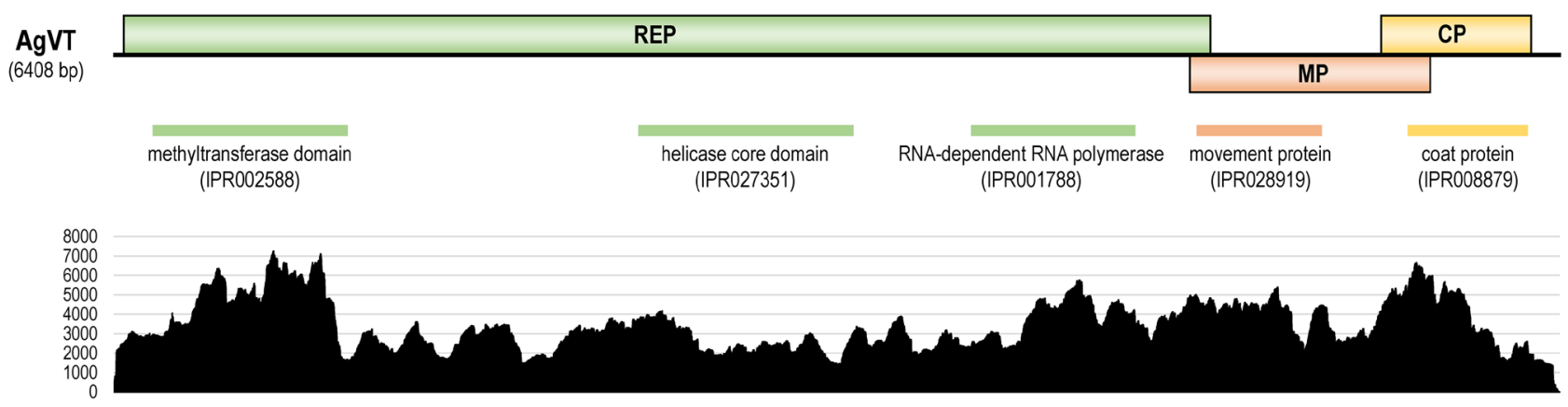

Fig. 1

Schematic representation of the AgVT genome organization

A schematic figure of the AgVT genome sequence is presented at the top. Three ORFs corresponding to the replicase (REP), the movement protein (MP), and the coat protein (CP) are depicted as boxes. Predicted InterPro domains are marked using lines below the ORFs with corresponding InterPro Acc. Nos. The sequencing depth is shown at the bottom. See Table 1 for the coordinates of the ORFs and InterPro domains.

(ChVT), one Ficus tepovirus A (FiTA), and one Trichosanthes tepovirus A (TrTA), were collected (Russo et al., 2009; Marais et al., 2015, 2020; Goh et al., 2019). Sequences of representative members of the other 12 Betaflexiviridae genera were also retrieved, including grapevine virus $A$ (GVA), Actinidia virus $B(A c V B)$, apple stem grooving virus A, Ribes americanum virus $A$, grapevine Pinot gris virus (GPGV), carrot Ch virus 1, Ocimum basilicum RNA virus 1 , citrus leaf blotch virus, apricot vein clearing associated virus, watermelon virus $A$, cherry rusty mottle associated virus, Phlox virus $B$, and apple stem pitting virus (Yoshikawa et al., 1992; Nakaune et al., 2008; Goh et al., 2018). Thirty-four known Betaflexiviridae virus genome sequences were retrieved for sequence comparison and phylogenetic analyses (Table 2).

Pairwise comparisons of protein sequences revealed that AgVT REP had 25.7-43.8\% aa identity with those of other Betaflexiviridae viruses. Four PVT REP sequences exhibited the highest similarity to AgVT REP sequence with 43.6-43.8\% aa identity. Other tepovirus REP sequences showed 32.3-33.1\% aa identity with AgVT REP sequence. REP sequences of viruses belonging to other
Betaflexiviridae genera showed 24.7-30.6\% aa identity with AgVT REP sequence. Comparison between the REP protein sequences confirmed that PVT was the most closely related to AgVT, among the currently known tepoviruses.

Multiple alignments of 35 REP sequences from AgVT, 11 other tepovirus, and 23 other Betaflexiviridae virus genomes were generated (Supplementary Fig. S1). The phylogenetic position of AgVT within the family Betaflexiviridae was inferred using the neighbor-joining method (Fig. 2). The subfamily Quinvirinae was used as the outgroup. AgVT was placed as a sister taxon of the four PVT sequences within the genus Tepovirus in accordance with the sequence comparison results. Among the genera of the subfamily Trivirinae, the genus Vitivirus was inferred to be the sister genus of Tepovirus.

PVT and PrVT are two currently approved species of the genus Tepovirus (https://talk.ictvonline.org, last accessed on December 5, 2020). According to the phylogenetic analysis, 12 REP sequences from seven tepoviruses were classified into two subclades: "PVT-clade" and "PrVT-clade." AgVT belonged to "PVT-clade" as the second member after PVT, which is the type species and found-

Table 1. ORFs of AgVT genome sequence

\begin{tabular}{|c|c|c|c|c|c|}
\hline ORF & $\begin{array}{c}\text { ORF } \\
\text { position (nt) }\end{array}$ & $\begin{array}{c}\text { Protein } \\
\text { length (aa) }\end{array}$ & $\begin{array}{c}\text { Domain } \\
\text { position (aa) }\end{array}$ & InterPro domain name & $\begin{array}{l}\text { InterPro } \\
\text { Acc. No. }\end{array}$ \\
\hline \multirow[t]{3}{*}{ Replicase (REP) } & $43-4860$ & 1605 & $43-329$ & alphavirus-like methyltransferase (MT) domain & IPR002588 \\
\hline & & & $761-1077$ & (+) RNA virus helicase core domain & IPR027351 \\
\hline & & & $1252-1493$ & tymovirus, RNA-dependent RNA polymerase & IPR001788 \\
\hline Movement protein (MP) & $4769-5836$ & 355 & $11-194$ & viral movement protein & IPR028919 \\
\hline Coat protein $(\mathrm{CP})$ & $5616-6278$ & 220 & $40-215$ & coat protein, trichovirus/vitivirus & IPR008879 \\
\hline
\end{tabular}


Table 2. Sequence comparison of the REP of AgVT and representative Betaflexiviridae viruses

\begin{tabular}{|c|c|c|c|c|c|c|}
\hline No & Subfamily & Genus & Virus & Acronym & NCBI & Identity $^{\mathrm{a}}$ \\
\hline 1 & Trivirinae & Tepovirus & Potato virus $\mathrm{T}$ & PVT & YP_002019748.1 & $723 / 1657(43.6 \%)$ \\
\hline 2 & & & Potato virus $\mathrm{T}$ & PVT & ADX41471.1 & $724 / 1657(43.7 \%)$ \\
\hline 3 & & & Potato virus $\mathrm{T}$ & PVT & AFU55321.1 & $723 / 1649(43.8 \%)$ \\
\hline 4 & & & Potato virus $\mathrm{T}$ & PVT & AXK90539.1 & $723 / 1652(43.8 \%)$ \\
\hline 5 & & & Prunus virus $\mathrm{T}$ & PrVT & YP_009051684.1 & $620 / 1881(33.0 \%)$ \\
\hline 6 & & & Prunus virus $\mathrm{T}$ & PrVT & AHM92766.1 & $620 / 1875(33.1 \%)$ \\
\hline 7 & & & Zostera virus $\mathrm{T}$ & ZoVT & QBS17025.1 & $621 / 1905(32.6 \%)$ \\
\hline 8 & & & Zostera virus $\mathrm{T}$ & ZoVT & QBS17031.1 & $616 / 1904(32.4 \%)$ \\
\hline 9 & & & Cherry virus $\mathrm{T}$ & ChVT & QNG41875.1 & $618 / 1893(32.6 \%)$ \\
\hline 10 & & & Ficus tepovirus A & FiTA & QED42804.1 & $605 / 1874(32.3 \%)$ \\
\hline 11 & & & Trichosanthes tepovirus A & $\operatorname{TrTA}$ & QED42832.1 & $607 / 1865(32.5 \%)$ \\
\hline 12 & & Vitivirus & Grapevine virus A & GVA & NP_619662.1 & $560 / 1830(30.6 \%)$ \\
\hline 13 & & & Actinidia virus B & $\mathrm{AcVB}$ & YP_004935358.1 & $531 / 1813(29.3 \%)$ \\
\hline 14 & & Capillovirus & Apple stem grooving virus & ASGV & NP_044335.1 & $536 / 1785(30.0 \%)^{b}$ \\
\hline 15 & & & Yacon virus A & YaVA & YP_009268859.1 & $524 / 1784(29.4 \%)^{b}$ \\
\hline 16 & & Ravavirus & Ribes americanum virus A & RAVA & YP_009553496.1 & $514 / 1971(26.1 \%)$ \\
\hline 17 & & Trichovirus & Apple chlorotic leaf spot virus & ACLSV & NP_040551.1 & $568 / 1976(28.7 \%)$ \\
\hline 18 & & & Grapevine Pinot gris virus & GPGV & YP_004732978.2 & $551 / 1937(28.4 \%)$ \\
\hline 19 & & Chordovirus & Carrot $\mathrm{Ch}$ virus 1 & $\mathrm{CtChV}-1$ & YP_009103999.1 & $572 / 1959(29.2 \%)$ \\
\hline 20 & & & Carrot $\mathrm{Ch}$ virus 2 & CtChV-2 & YP_009103996.1 & $568 / 1946(29.2 \%)$ \\
\hline 21 & & Divavirus & Diuris virus A & DiVA & YP_006905850.1 & $552 / 1857(29.7 \%)^{\mathrm{b}}$ \\
\hline 22 & & & Ocimum basilicum RNA virus 1 & ObRV1 & YP_009408144.1 & $547 / 1837(29.8 \%)^{\mathrm{b}}$ \\
\hline 23 & & Citrivirus & Citrus leaf blotch virus & CLBV & NP_624333.1 & $581 / 2042(28.5 \%)$ \\
\hline 24 & & & Citrus leaf blotch virus & CLBV & AFA43536.1 & $565 / 2080(27.2 \%)$ \\
\hline 25 & & Prunevirus & Apricot vein clearing associated virus & AVCaV & YP_008997790.1 & $532 / 1825(29.2 \%)$ \\
\hline 26 & & & Caucasus prunus virus & $\mathrm{CPrV}$ & YP_009505632.1 & $569 / 2121(26.8 \%)$ \\
\hline 27 & & Wamavirus & Watermelon virus A & WVA & YP_009357235.1 & $552 / 1907(28.9 \%)$ \\
\hline 28 & & & Watermelon virus A & WVA & QEA69426.1 & $558 / 1922(29.0 \%)$ \\
\hline 29 & Quinvirinae & Robigovirus & Cherry rusty mottle associated virus & CRMaV & YP_007761581.1 & $548 / 2121(25.8 \%)$ \\
\hline 30 & & & Cherry twisted leaf associated virus & CTLaV & YP_009046478.1 & $560 / 2163(25.9 \%)$ \\
\hline 31 & & Carlavirus & Phlox virus B & PhlVB & YP_001552317.1 & $554 / 2155(25.7 \%)$ \\
\hline 32 & & & Garlic common latent virus & GCLV & YP_004936159.1 & $544 / 2060(26.4 \%)$ \\
\hline 33 & & Foveavirus & Apple stem pitting virus & ASPV & NP_604464.1 & $564 / 2279(24.7 \%)$ \\
\hline 34 & & & Asian prunus virus 1 & APV1 & YP_009094347.1 & $572 / 2112(27.1 \%)$ \\
\hline
\end{tabular}

aProtein sequence identity in the form of "number of identical residues/aligned length (percent identity).” $\mathrm{CP}$ regions were removed from the capillovirus and divavirus REP-CP protein sequences.

ing member of the genus Tepovirus. Bootstrap analysis strongly supported the placement of both subclades with $100 \%$ bootstrap values. However, the Tepovirus clade, and hence the monophyletic origin of "PVT-clade" and "PrVTclade" viruses, was only marginally supported with a bootstrap value of $72.1 \%$. This suggested the possibility that "PrVT-clade" might be classified as a novel genus different from "PVT-clade."

The monophyletic relationship between "PVT-clade" and "PrVT-clade" was further investigated using MP and $\mathrm{CP}$ sequences of all tepoviruses, two vitiviruses (GVA and AcVB), and a trichovirus (GPGV). Multiple alignments of 


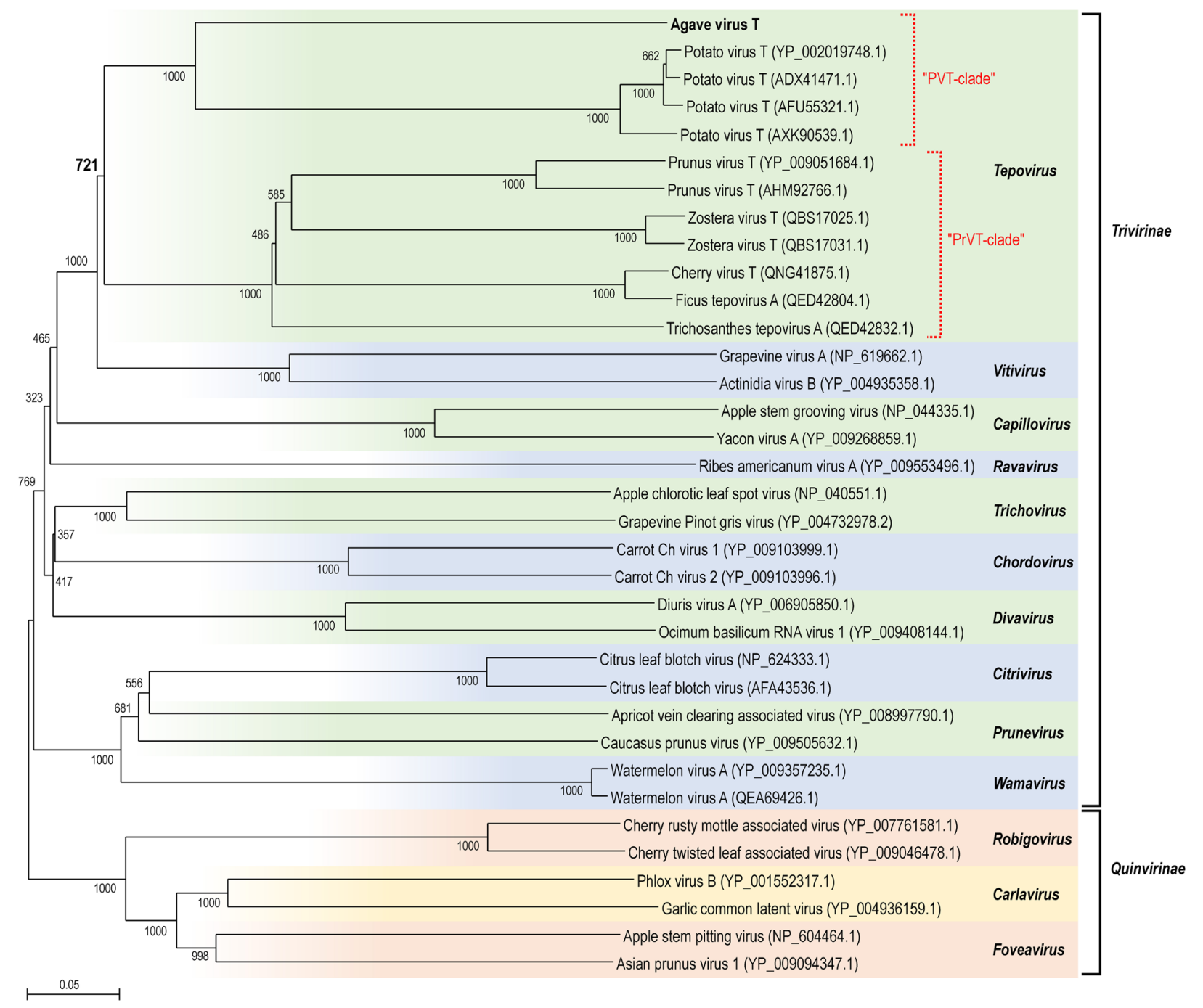

Fig. 2

Phylogenetic position of AgVT among Betaflexiviridae viruses

A phylogenetic tree was constructed based on REP sequences of AgVT, previously known tepoviruses, and representative members of the family Betaflexiviridae using the neighbor-joining method. Tepoviruses can be classified into two subclades: "PVT-clade" and "PrVTclade." AgVT is the second member of "PVT-clade." The subfamily Quinvirinae was used as the outgroup. The NCBI protein sequence Acc. Nos. are in parentheses. Bootstrap supporting values calculated from 1000 replicates are shown at the nodes. The bootstrap value for the Tepovirus clade is highlighted in bold.

MP and CP sequences were generated (Supplementary Figs. S2 and S3, respectively). Phylogenetic trees of MP and $C P$ sequences were constructed using the neighborjoining method (Fig. 3). In each tree, the sequence of GPGV was used as the outgroup. In both trees, the monophyletic origin of all 12 tepovirus sequences was represented. The monophyletic relationship of "PVT-clade" and "PrVTclade" was strongly supported in the MP tree with a bootstrap value of $94.4 \%$. However, in the CP tree, the placement of the Tepovirus clade was very weakly supported with a bootstrap value of $57.2 \%$. These results indicate the need for performing more comprehensive phylogenetic analyses to clearly understand the relationship between "PVT-clade" and "PrVT-clade" within the genus Tepovirus as well as within the family Betaflexiviridae.

In conclusion, the genome sequence of AgVT, a putative novel member of the genus Tepovirus of the family Betaflexiviridae, was identified from transcriptome data obtained from the stem sample of a blue agave plant. Sequence comparison and phylogenetic analyses indicated that AgVT was the closest relative of PVT among all currently known tepoviruses, thereby forming a distinct 
(a) MP

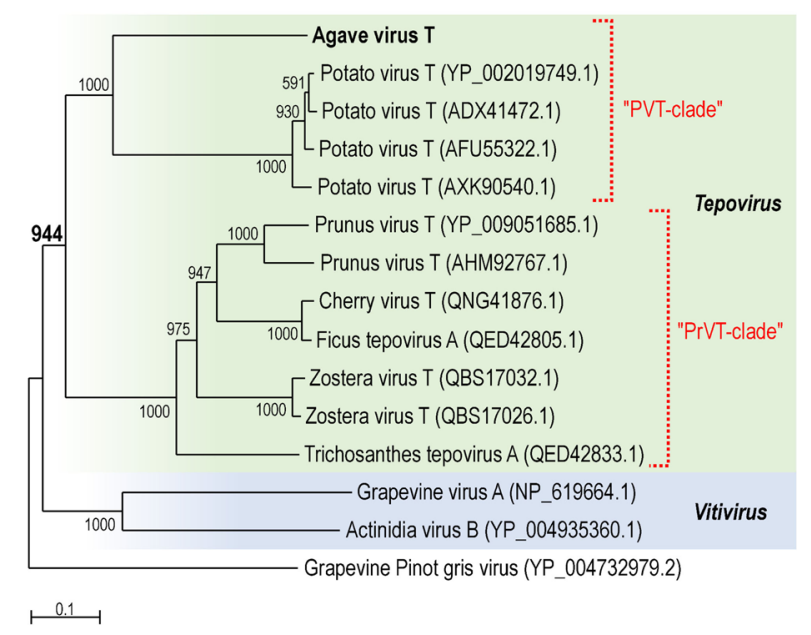

(b) CP

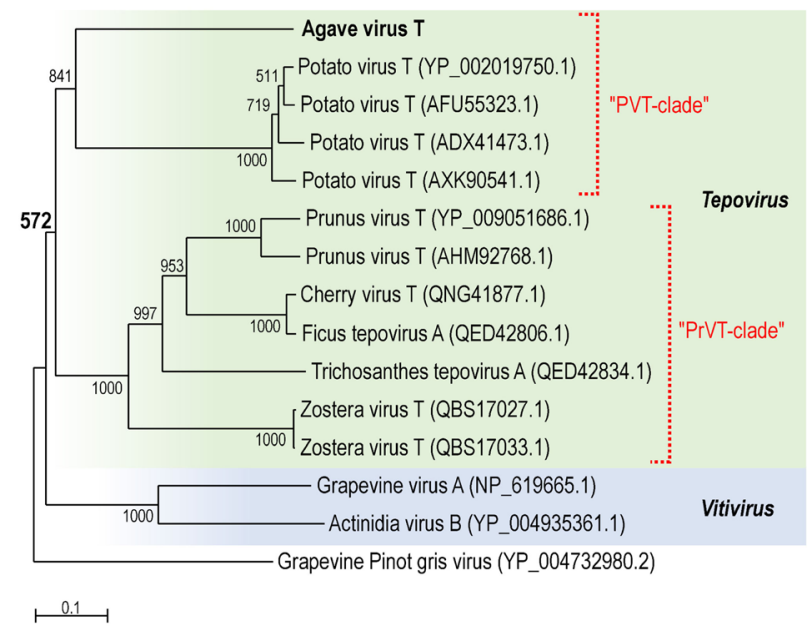

Fig. 3

Phylogenetic relationships of tepoviruses inferred from MP and CP sequences

Neighbor-joining phylogenetic trees were inferred from MP (a) and CP (b) sequences of tepoviruses, two vitiviruses, and a trichovirus. Two subclades ("PVT-clade" and "PrVT-clade") were reproduced within the genus Tepovirus. The sequence from the grapevine Pinot gris virus, a trichovirus, was used as the outgroup. The NCBI protein sequence Acc. Nos. are in parentheses. Bootstrap supporting values calculated from 1000 replicates are presented at the nodes. The bootstrap value for the Tepovirus clade is indicated in bold.

subclade. The AgVT genome sequence may be useful for studying the phylogenetic relationships of tepoviruses and other closely related Betaflexiviridae viruses.

Acknowledgments. This work was supported by the National Research Foundation of Korea (NRF) funded by the Government of Korea (grant Nos.2018R1A5A1025077 and 2020R1A2C1013403).

Supplementary information is available in the online version of the paper.

\section{References}

Adams MJ, Candresse T, Hammond J, Kreuze JF, Martelli GP, Namba S, Pearson MN, Ryu KH, Saldarelli P, N.Y (2012): Family - Betaflexiviridae. In King AMQ, Adams MJ, Carstens EB, Lefkowitz EJ (Eds): Virus Taxonomy: Classification and Nomenclature of Viruses. Ninth Report of the International Committee on Taxonomy of Viruses. Elsevier, San Diego, pp. 920-941. https://doi. org/10.1016/B978-0-12-384684-6.00078-1

Borland AM, Griffiths H, Hartwell J, Smith JA (2009): Exploiting the potential of plants with crassulacean acid metabolism for bioenergy production on marginal lands. J. Exp. Bot. 60, 2879-2896. https://doi.org/10.1093/jxb/ erp118

Cedeno M (1995): Tequila production. Crit. Rev. Biotechnol. 15, 1-11. https://doi.org/10.3109/07388559509150529
Coleman-Derr D, Desgarennes D, Fonseca-Garcia C, Gross S, Clingenpeel S, Woyke T, North G, Visel A, PartidaMartinez LP, Tringe SG (2016): Plant compartment and biogeography affect microbiome composition in cultivated and native Agave species. New Phytol. 209, 798-811. https://doi.org/10.1111/nph.13697

Goh CJ, Park D, Hahn Y (2020): Identification of Trichosanthes associated rhabdovirus 1 , a novel member of the genus Cytorhabdovirus of the family Rhabdoviridae, in the Trichosanthes kirilowii transcriptome. Acta Virol. 64, 36-43. https://doi.org/10.4149/av_2020_105

Goh CJ, Park D, Kim H, Sebastiani F, Hahn Y (2018): Novel Divavirus (the family Betaflexiviridae) and Mitovirus (the family Narnaviridae) species identified in basil (Ocimum basilicum). Acta Virol. 62, 304-309. https:// doi.org/10.4149/av_2018_224

Goh CJ, Park D, Lee JS, Davey PA, Pernice M, Ralph PJ, Hahn Y (2019):Zostera virus T - a novel virus of the genus Tepovirus identified in the eelgrass, Zostera muelleri. Acta Virol.63,366-372.https://doi.org/10.4149/av_2019_409

Gross SM, Martin JA, Simpson J, Abraham-Juarez MJ, Wang Z, Visel A (2013): De novo transcriptome assembly of drought tolerant CAM plants, Agave deserti and Agave tequilana. BMC Genomics 14, 563. https://doi. org/10.1186/1471-2164-14-563

Huang X, Wang B, Xi J, Zhang Y, He C, Zheng J, Gao J, Chen H, Zhang S, Wu W, Liang Y, Yi K (2018): Transcriptome comparison reveals distinct selection patterns in domesticated and wild Agave species, the important CAM plants. Int. J. Genomics 2018, 5716518. https://doi. org $/ 10.1155 / 2018 / 5716518$ 
Kim H, Park D, Hahn Y (2018): Identification of novel RNA viruses in alfalfa (Medicago sativa): an Alphapartitivirus, a Deltapartitivirus, and a Marafivirus. Gene 638, 7-12. https://doi.org/10.1016/i.gene.2017.09.069

Marais A, Faure C, Mustafayev E, Barone M, Alioto D, Candresse T (2015): Characterization by deep sequencing of Prunus virus T, a novel tepovirus infecting Prunus species. Phytopathology 105, 135-140. https://doi.org/10.1094/ PHYTO-04-14-0125-R

Marais A, Šafářová D, Navrátil M, Faure C, Cornaggia D, Brans Y, Suchá J, Candresse T (2020): Complete genome sequence of cherry virus T, a novel cherry-infecting tepovirus. Arch. Virol.165,1711-1714. https://doi.org/10.1007/ s00705-020-04656-w

Martelli GP, Minafra A, Saldarelli P (1997): Vitivirus, a new genus of plant viruses. Arch. Virol.142,1929-1932. https://doi. org/10.1007/s007050050088

Morozov SY, Solovyev AG (2003): Triple gene block: modular design of a multifunctional machine for plant virus movement. J. Gen. Virol. 84, 1351-1366. https://doi. org/10.1099/vir.0.18922-0

Nakaune R, Toda S, Mochizuki M, Nakano M (2008): Identification and characterization of a new vitivirus from grapevine. Arch. Virol. 153, 1827-1832. https://doi. org/10.1007/s00705-008-0188-5

Nibert ML, Pyle JD, Firth AE (2016): A + 1 ribosomal frameshifting motif prevalent among plant amalgaviruses. Virology 498,201-208. https://doi.org/10.1016/j.virol.2016.07.002

Park D, Goh CJ, Kim H, Hahn Y (2018): Identification of two novel amalgaviruses in the common eelgrass (Zostera marina) and in silico analysis of the amalgavirus +1 programmed ribosomal frameshifting sites. Plant Pathol. J. 34, 150-156.

Park D, Goh CJ, Lee JS, Sebastiani F, Hahn Y (2020): Identification of Pistacia-associated flexivirus 1, a putative mycovirus of the family Gammaflexiviridae, in the mastic tree (Pistacia lentiscus) transcriptome. Acta Virol. 64, 28-35. https://doi.org/10.4149/av_2020 104

Prosser SW, Xiao H, Li C, Nelson RS, Meng B (2015): Subcellular localization and membrane association of the replicase protein of grapevine rupestris stem pittingassociated virus, family Betaflexiviridae. J. Gen. Virol. 96, 921-932. https://doi.org/10.1099/igv.0.000019

Rubino L, Russo M, De Stradis A, Martelli GP (2012): Tepovirus, a novel genus in the family Betaflexiviridae. Arch. Virol. 157, 1629-1633. https://doi.org/10.1007/s00705012-1342-7

Russo M, Rubino L, De Stradis A, Martelli GP (2009): The complete nucleotide sequence of potato virus T. Arch. Virol. 154, 321-325. https://doi.org/10.1007/s00705008-0300-x

Veerakone S, Liefting LW, Tang J, Ward LI (2018): The complete nucleotide sequence and genome organisation of a novel member of the family Betaflexiviridae from Actinidia chinensis. Arch. Virol.163,1367-1370.https:// doi.org/10.1007/s00705-017-3701-x

Yoshikawa N, Sasaki E, Kato M, Takahashi T (1992): The nucleotide sequence of apple stem grooving capillovirus genome. Virology 191, 98-105. https://doi.org/ 10.1016/0042-6822(92)90170-T 


\title{
A novel tepovirus, Agave virus T, identified by the analysis of the transcriptome data of blue agave (Agave tequilana)
}

\author{
Chul Jun Goh, Dongbin Park, Yoonsoo Hahn* \\ Department of Life Science, Chung-Ang University, Seoul 06974, Republic of Korea
}

Received January 8, 2021; accepted January 19, 2021

\begin{abstract}
Summary. - The genome sequence of a novel RNA virus was identified by analyzing transcriptome data obtained from the stem sample of a blue agave (Agave tequilana) plant. Sequence comparison and phylogenetic analysis showed that the RNA virus, Agave virus T (AgVT), was a new member of the genus Tepovirus in the family Betaflexiviridae. AgVT genome had three open reading frames: a 1605 -amino acid (aa) replicase (REP), 355-aa movement protein (MP), and 220-aa coat protein (CP). Phylogenetic analyses based on the REP, MP, and CP sequences of AgVT, previously reported tepoviruses, and other Betaflexiviridae viruses revealed that tepoviruses could be classified into two subclades: "potato virus T(PVT)-clade" and "Prunus virus T (PrVT)-clade." PVT, the type species and founding member of the genus Tepovirus, belong to "PVT-clade" along with AgVT, while the other five tepoviruses belong to "PrVT-clade." The genome sequence of AgVT may be useful for studying the phylogenetic relationships between tepoviruses and other closely related viruses.
\end{abstract}

Keywords: Agave virus T; Tepovirus; Betaflexiviridae; blue agave; Agave tequilana 
Fig. S1. Alignment of replicase sequences of AgVT and related viruses

AgVT
PVT:YP_002019748.1 PVT :ADX̄41471.1 PVT:AFU55321.1 PrVT:YP_009051684.1 PrVT:AHMM92766.1 ZOVT: QBSS17025.1 ZoVT:QBS17031.1 ChVT: QNG41875.1
FiTA:OED42804.1 TrTA:QED42832.1 ACVB: $Y \bar{P}_{-} 004935358.1$ ASGV:NP-044335.1 YaVA:YP- 009268859.1 ACLSV:N $\bar{P}$. 040551.1 GPGV:YP_-004732978.2 CtChV-1:YP_009103999.1
CtChV-2:YP 009103996.1 DiVA:YP 006905850.1 ObRV1:YP CLBV: NP 624333.1
CLBV: AFA443536.1 AVCaV:YP 008997790.1 WVA:YP 009357235.1 WVA: QEAĀA9426.1
CRMaV:YP_ 00771581.1 CTLaV:YY-009046478.1 \begin{tabular}{l} 
PhIVB:YP \\
GCLV:YP 001552317.1 \\
\hline
\end{tabular} ASPV:NP-604464.1.1 APV1:YP_-009094347.1

AgVT
PVT:YP_002019748.1 PVT: ADX̄ 41471.1 PVT : AFU55321.1
PVT : AXK90539.1 PrVT:YP_OO9051684.1 PrVT: AHM992766.1
ZoVT:OBS17025.1 ZOVT:QBS17031. ChVT:QNG41875.1
FiTA:QED42804.1 TrTA:QED42832.1 ACVB:YP_ 004935358.1 ASGV:NP- 844335.1 YaVA:YP-
RAVA:YP
-0095553496.1 ACLSV:NP 040551.1
GPGV:YP $\overline{0} 04732978.2$ CtChV-1:YYPOO9103999.1 CtChV-2:YP 009103996.1
DiVA:YY 006905850.1 DiVA:YP 0 ObRV CLBV:NP 624333. AVCaV:YP 008997790.1 CPrV:YY $\overline{0} 009505632.1$ WVA:YP CRMaV:YP_ 007761581.1 CTLaV:YP-009046478.1 PhIVB:YP 001552317.1
GCLV:YP_004936159.1 ASPV:NP-684464.1.

AgVT
PVT:YP_002019748.1 PVT: ADD X 41471.1
PVT: AFU55321.1 PVT: AXK90539.1 PrVT:YP 009051684. PrVT: AHM92766.1
ZOVT: QBS17025.1 ZOVT:QBS17031.1 ChVT: ONG41875.1 FiTA:QED42804. GVA :NP 619662.1
ACVB:Y ACVB: $\overline{\text { P }}$ 0044935358.1 ASGV:NP-044335.1 YaVA:YP- 009268859.1
RAVA:YP 009553496.1 ACLSV:ND 0045551.1 CPChV - Y CtChV-2:YP-009103996.1 DiVA:YP. CLBV:NP -624333.1 AVCaV:YP 008997790.1 CPrV:YP $\overline{0} 09505632.1$ WVA: QEĀ 69426.1 CRMaV:YP 007761581.1 PhIVB:YP-001552317.1 GCLV:YP_004936159.1 APV1:YP_-009094347.1

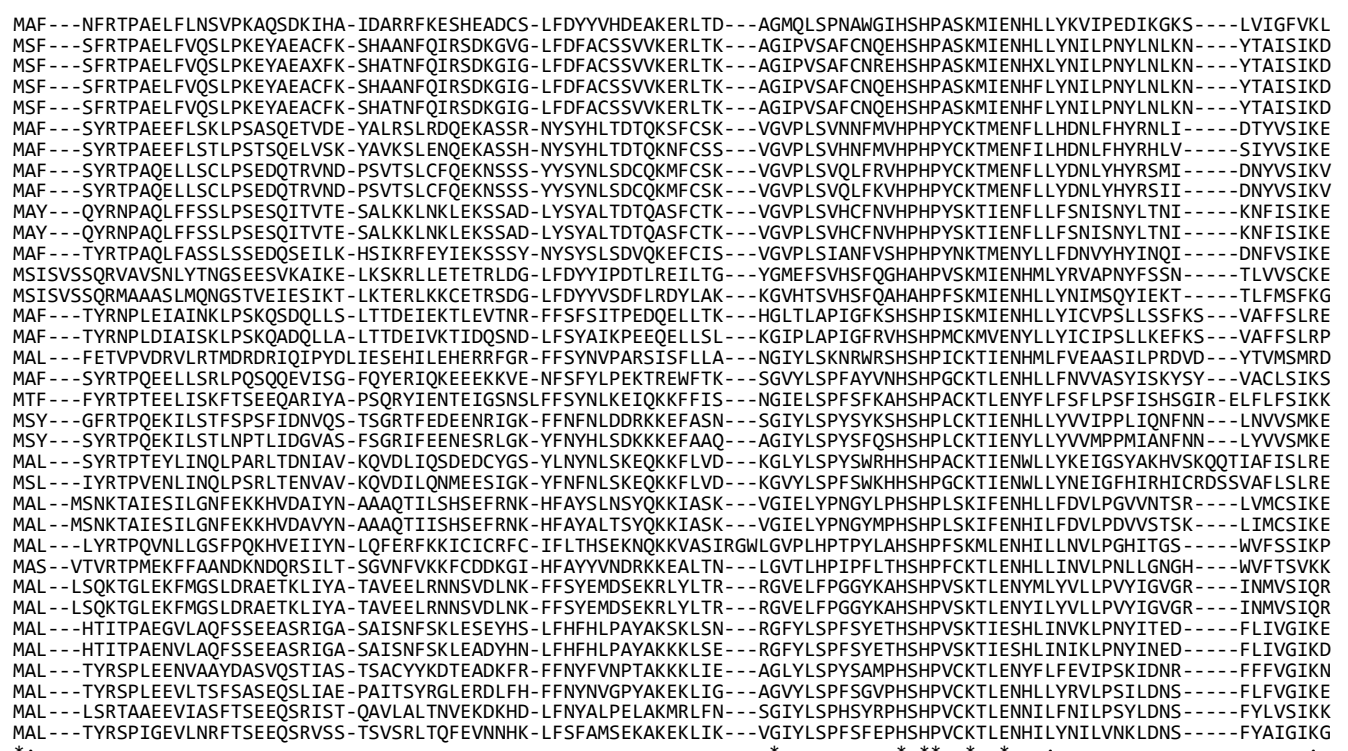

SKLDKFLSRNK- 10.

SKLDKFLSRNK- 10. SKVRKLLKNGV--. EKVGLLNRSRG-1.-1 DKVNLLKRGGH DKVNLLKRGGH-RNGFVIINRVIADRDVLRYGPCGYNKE -FENKNNFIPNQ- - -SKSWFFHDELHH -FMCEDFLSGEKNSFHRLINRIIADRDKLRYSTELKVQNN SLAQIGGI----DESWFFHDELH NQIKQIVDGRK----NERWFFHDELHH -NLTNLINKEDQ----SECIFIHDEVQ - HLPELARLSET----CETAVIHDEVQY LRDQCLTRNKF---PKVLFLHDELHF LRDDCLRANKF--- PKVLFLHDEVHF -VSPERIEKRKT - - -GLDIFIHDEIHH ESNT -RDYDPLMDVRIEK - GSNFLFHDELHY SFDKIGDQIGP---RASFYIHDECHY SKVLVFKGIRDKSRRQVSDLNAL---NSLNNSHTSF INRLVASKDVSRYTEEADAFFQS--
SKVLIFKNIRDRKK---DNGALGFCGKDTSASDHTSFVNRLVAPKDIRRYTEEADAFFSSK-

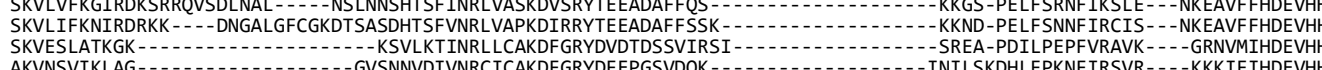
SKIKRLRLKNANKVDSFLKMHS-1--- SHFKIKSLGMYNAIIDGKDKYRYGDVEFSSFRDRVIG SKFSALKIARS NKMSKMERLGP - NVQLNHLNRLVEVKDKMRYGMD APKKMSINLINRLMDVKDSFRYKSGDGLSIKYPTQLL GKLNAIKKIHFEKKN-
GKLKNLEKIHFRQGN-AKVNSVIKLAG-1-1 KKVLNLSLKMN-1 NKLSVLRKDKK-NKLSVLRKEKK-EKLSMLKARNT SKLNFLRARHQ--
NKVDFLKRRHP-SKVNFLKSRSK-- - - -

WSLDQLNELLEN-SSLDFLVATVVHPFEVEMGEKKSLNPELYNFKV-KGDK WSMNSLSDFLDR-SNVKELLATIVFPIEILLGSKRSLNPELYEFEI -SRGKWSMNSLSDFLDR-SNVKELLATIVFPIEILLGSKRSLNPELYEFI -SRGK

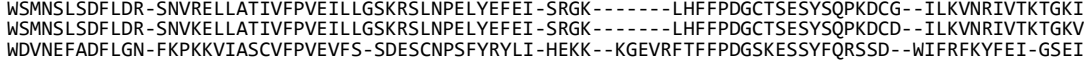

FNYPNGKVEEAYVQPKHLP- -LHFISEFIN-KRGR-- - - -FAVERTYSLGAHHVF -LHFFPDGCTSESYSQPKDCD- - ILKVNRIVTKTGKI- - - FSVELIHTIGANHM LHFFPDGCTSESYSQPKDCG--ILKVNRIVTKTGKI SVELIHTIGANHM SVELVHTIGANHMI WNLEDLTKFIKR-ANPERMLATLVIPPEIFQ-LKNSMNPSFYDYET

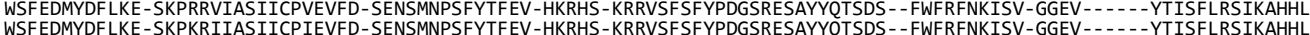

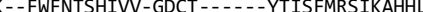
WSLDEMORFLGSLSKVDRWYSIIYPSEVEAGYSQSLFPEAYTFDL-KDRR - - - - LVWYPDGKAEGAYTOPVNP - -WLLRCSKTEDSKGRS - - - - WTITKLOTIGAHHL

WSLQDFQLVLGQMNKVKRLMYSIIYPAE IDQGYEHSLFPEAYHFER -RGGY-- - - FIWLPDGQSDGAYKQPINP --WLLSTSKTIDSRGRT- - - WTIGKVTSFASHHLF LSPFDMAFLFETIPEIDRVVATTVFPIELLFGDKVSKEPRVYTYKV-HGSS- -1 . LTPFDIAYLFETIPEIDRVVATSVFPPELLMGDRVSKEPRVYSFKV-RGDD WSKRQMFYFLDS-VSPENFYFTAVYPTEVLAGLDYSLYPSVYQFTL - KGDK
WSRDOLETFLOV-HRPKNLWATLVFPPETLAGYKSSVLPFLYOFEI - HGKD WSKAQLISFLEV-HRPRNIMATVVFPIEILGGFKSSVLKFLYEFEC-RNGK WTFSMMLDFLEK-FEPSHVICTAVFPVEILEGIKOSLYPEVYSFEILQTGN WSFDMMLDFLRK -FEPDNVLCTVIFPVEIFSGVKQSLFPDVYSFQIIKNKR WSPNDLSNFLSR-TKAESILATVIHPTEIDVGKDCSHLPFLYEFEV-SDNN
WNPADLDRFIRY-TDAESIMFTVIHPVEVDVGKSSHLPFLYEFCI-DGET WTKAQMFSFLKS - TKVKRFIFTVVYPPEILKKFANSQNPKVYDFKV-DKGR WTKAQMFSFLKR-TKVRRFIFTIVYPPELLKKFANSQNPKVYDFKV-DKGR WTLDDMLGFLDR-ARPNRFVFSVVYPVELLAGILESQNPKMYKFQDSKSDK WSHLNMIQF LEE - TATPLLLCSVVFPPELLGGIKTPQNSALYGFQV-DGDK
WSLNDITYF IEE -VKPKRMLVSVVYPPELLLGIESSLNKLAYDFEINDDGT WSLNDITYFIEE -VKPKRMLVSVVYPPELLLGIESSLNKLAYDFEINDDGT
WSLNDITYFIEE-VKPKRMLVSVVYPPELLLGIESSLNKLAYDFEINDDGT WSLNDITYFIEE-VKPKRMLVSVVYPPELLLGIESSLNKLAYDFEINDDGT
WSMKDIVDFLEI-SKAKTIIGSFVFPSEILAGARTSLNPWAYEFKI -KGDK WSARDLITFLEV-IQPEVMYATMVYPPELLGSKFSLFKWCYEFDV - QGNDD-WCERDLVTFLSA-VKPEKVLGTVWYPPELLKGVKFSLNKWCYDFDV IGND-WTKEALITFLDH-VKPEVMLASIVFPPEILAGAKESLNPWCYTFRI-VGKD WSSHDLMLFLDS-INPDHLLATIVFPPEILAGAQESLNPWCYSFQR-HGSK

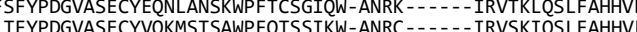

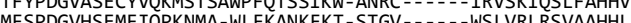

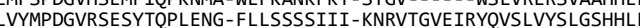
作 作 VSVTLLRTIGPFHLI YNVTLLRSIGPFHLM

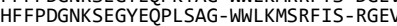

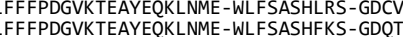
IVFFPDGRASEGYEQRANLR-WLFCASHFRT-SGSI LFFFPGSRSEMYEQPSNLN-WLFEASYIHT-SAGT FSFYPDGVKTEAYNQRVNLD-WLMKGSYLRT-NNGV TFYPDGVRTESYTOPISGC-FYLKVGTLLPLGSV源 LTFFPDGVQSESYTQPLSAG-YILQASKIVCPSGQV LFYYPDGVMSEAYVQTLESS-YLFKTNLIKT-SKGH--.--YSVSLHRSVGSHHF FVFPDGCYSESYEOSVDMK-WLFSAASFVV-DDLL-IYAPDGVWSESYEQPLSAG-QLLKFNKIMT-RNGS 
AgVT
PVT:
PFP

PVT:YP 002019748.1

PVT:ADX41471.1

PVT:AXK90539.1

PrVT:YP 009051684.1

PrVT: AHM̄M2766.1

ZoVT:QBS17025.1

ChVT: QNG41875.1

FiTA:QED42804.

GVA:NP 619662.1

ACVB: $Y \bar{P}-004935358.1$

ASGV:NP- 044335.1 .

YaVA:YP- 009268859.1
RAVA:YP 00953496.1

RALA:YP

GPGV:YP_-̋04732978.

CtChV-1:YY
CtChV-2:YP Y09103999.1

DiVA:YP 006905850.1

CLBV:NP 624333.1

CLBV:AFA A 43536.1

AVCVV:YP 008997790.1

CPrV:YP
WVA: YP 009505632.1
DVA

WVA:QEĀ 69426.1

CRMaV:YP_007761581.1

CTLaV:YY-009046478.1

GCLV:YP_ö04936159.1

APV1:YP ${ }^{\circ} 009094347.1$

AgVT
PVT :YP 002019748.1

PVT: $A D \bar{X} 41471.1$

PVT :AFU55321.1
PVT: AXK90539.1

PVT:AXK90539.1
PRVT:YP 009051684.1

PrVT:AHM̄92766.1

ZoVT:QBS177025.

CVVT:QNG41875

FiTA:OED42804.

TrTA: OED 42832.1

ACVB: $Y \bar{P}$ - 0049353558.1

ASGV:NP- 044335.1

YaVA:YP-
RAVA:YP 0092688595.1
009596.1

ACLSV:NP 040551.1

CtChV-1-YP 009103999

CtChV-2:YP-009103996.1

DiVA:YP $00 \overline{6} 905850.1$

ObRV1:YP -009408144.1

CLBV:NP- 624333.1

AVCaV:YP 008997790.1

WV:YP

WVA:QEĀ 69426.1

CRMaV:YP_-007761581.1

CTLaV:YP-009046478.1

GCLV:YP 004936459.1

ASV:ND-604464.1
APV1:YP- 099094347.1

AgVT
PVT:YP_Q02019748.1

PVT :AD̄ 41471.1

PVT : AFU55321.1
PVT: AXK90539.1

PrVT:YP_009051684.1

PrVT: AHM̄M2766.1

ZoVT:QBS17025. 1

ChVT:ONG41875.

FiTA:QED42804.

TrTA: QED42832.1

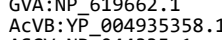

Y

RAVA:YP 092553496.1

ACLSV:NP 040551.1

GPGV:YP

CtChV-1:YP-O09103999.1
CtChV-2:YP 2009103996.1
DiVA YP 00695850.1

DiVA:YP $00 \overline{6} 905850.1$
ObRV1:YP 009408144.1

CLBV:NP_624333.1

CLBV: AFA43536.1
AVCaV:YP 008997790.1

CPrV:YP -009505632.1

WVA:QEÄ69426.1

CRMaV:YP_007761581.1

CTLaV:YY-009946478.1

PhIVB:YP 001552317.1

GCLV:YP $\bar{\theta} 044363515.1$

ASPV:NP_604464.1
APV1:YP_09094347.1
VIQRGC-LLTQSQQVAPSTSAMSFSWLLG---FGTPGAVRVRINIFKKLITYLMSLKKPDRESAVAKVRQLCNSEIF TDELLFAVHLGKLIAEMGM-DDNFGRQGIVKFLVRGLA MIKEGS-FDVDSERFFDRSSALTTSLLMPTR---AGKALRIRRKFLLRLIIYLFSLKKPDHHSAIAKIRQSSDDSIFCDEIMLADHVGKIFEKLDP-ASPFGVKGVFLLTSIFK MIKEGS-FVDSERFFDRSSALTTSLLMPTR---AGKALRIRRKFLLRLIIYLFSLKKPDHHSAIAKIRQSSDDSIFCDEIMLADHVGKIFEKLP -ASPFGVKGVLDLLTGIFK MIKEGS-FDVDSERFFDRSSALTTSLLMPTR---AGKALRIRRKFLLRLIIYLFSLKKPDHHSAIAKIRQSSDDSIFCDEIMLADHIGKIFEKLDP-ASPFGVKGVFDLLSGIFK LIEKGK-LETNDYYISDEAECASLRMFGTAV-AGK-ITAPIRVEVLKREMIYLLSLKKSDMNSAAAKLRQLSQEDYHPQELCFFLNLAGRIESV---KGIFKDRGLVSTVIDCFA
LIERGR-LETNRFFISDSAECASLKMFGTAV-AGK-ICAPIRVEVLKREMIYLLSLKKSDMNSAAAKLROLSOEDYHPOELCFFLNLAGRIESV---KGIFKDRGLVSTIMDCFA LIERGR-LETNRFF ISDSAECASLKMFGTAV-AGK-ICAPIRVEVLKREMIYLLSLKKSDMNSAAAKLRQLSQEDYHPQELCFFLNLAGRIESV---KGIFKDRGLVSTIMDCFA
LIEKGK-LKTNPFYVSDFAECINAKMIGFSR-ISH-ESIPIRELVFKREIIYLMSLKKPDMHSATAKLRMLSSEDYTTGELLFFNALACEIDSV---KGLHADISLLKHVQMAML 作 VEKGN-FETSPYYISDYSECISARLFGFTR-YN - -SFMPIRFEVLKKELIYLLSLKRCDPNSAAAKLROLSDEGYTGPELAFFLKLAGHIAKA- - - - KVFDDRTLFSCLYSNFC IOKGN-LKTSERYISDNSECLCSSVFDTSR - IKKRGL IPIRWKIFKSEIIYLMSLKKPDSOSAAAKLROLSDGDYTGGELAFFMNLAKKISNS - - -KKVFDCADLLPAIFDSII SAIKGS-YLTEESYKYDNFTIINPNDVLKGK -RGG-KPLYLRARMIKPTLLYLLALKKSDSNSAVAKLRMLSSREENMDEALFVAQLAKOIKDTAL - YDKMGNPNLRSILSESFY SFDRGR-ACNEFNH-FDKPSCLLAEEMRLLT-KRF-DKAVINRSTVSSLSTYMACLKTANAASAVAKLRQLEKRDLYPDDELNFVYSFGEHFKNFGM--RDDFDVSVLOWVKDKFC SFDRGK-GGSKLNH-FDKPSCLLAFELRTLT-KRF-NEAVVNRSVVSSLSTYMACLKTANAASAVAKLRQLEKRDLLPDELNFVYSFGDHFKNYGM--RNDLDITILQWIKDKIC

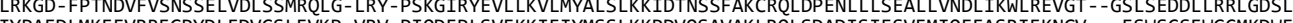

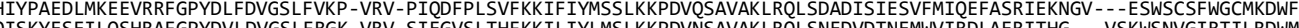
QISKYESETLQSHRAFGPYDVDVGSLFRGK-VRV-SIEGVSLTHFKKILIYLMSLKKPDVNSAVAKLRQSNED TIVKGR-RISRSIRLFKGFDTLDMSAFLGTK-YKM-PIADVHFSFFKKIVIYLKSLKKPDTQSAVAKLRQLVGDSISLTEVLFIEDFASRFQEHGA--AKLSNDGIFDQFIHSLK YISRGS-LASESRRFFDDFNILDLPVKYAKNNLIKKMKLLL RNNFMIKIVSYIKS KKPDKESAIAKLRML SEDEFSLEEMIFVDGLVDTLLNGY--KS TWENGWVEWFICGLK FLSRGG-RVVESKRFFTDFNIDLPSANLF EISIGE-LVTDSKLFFSDYNSIDMSKIFLDR-FRSYEVFPISIEHLYKVYSYLLCLKKPDLESGLAKLROIIGDDVEIKEFLFFEOFCKRLIEROT-SWGLFGHSFFEKLTDMAL EVSMGE-LISDSKIFFSDYGSIDMSKIFLDR - FRSYEVFPIAIEHLYKVYSYLLCLKRDLESGLAKLROIIGDDVE TKEFLFFEOFCKRLIEROT - SWGLFGYSFFDKLTDLAL EVVPGN-YFTDEIRFFNDFETIDLOCIFKSR-FLCRDFVPISKDLVERVYSYLICLKKPDMOSAMAKLKOLMGDDLDVRVOVFFRSLVHRILNESE -CFSLFDVSIVNKWKKKF QISKGE-KITDSVRFFADFNTIDMSVIHKER-FKYYDLIPIKKSHIEKIYTYLLCLKKPDVESAIAKLRQLMEDEQDCRVVEFFCTFAKKLITDTKGAINLFGDSFLQKAKDSF

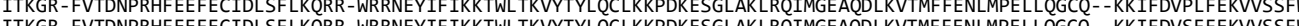
EITKGR-FVTDNPRHFEEFECIDLSFLKQRR-WRRNEYIF IKKTWLTKVYTYLQCLKKPDKESGLAKLRQIMGEAQDLKVTMFFENLMPELLQGCQ--KKIFDVSFFEKVVSSFW 作 IINRDN-LLNEEFRVYSEFDAVSIRRLNYLG-GNSDDIIPVRYEVILSVFKYIRTLKKPDLQSGMAKHRQLVDEPTGF-EVRFIEDFVQFILENHE-KFNLIGQKF SNFLSSAC SITAGE-AVPAPTNSFSNFDATTQRGP SIAGE-AVRAPTNSFSNFDATTCRGLPNIAFRSLGPCIAVPYPVISRVYRYLRTLQKPDQSAMAKLSQIMPEPTGY-QIKFMTEFSKMVDTPV-GASFFQVAIFEKFKGFL SITKGD-LPGLERRFFSNFEATGVKHLSGLS-YSVRDCIPVSSELISKLYRYIRTLKKPDLQSCMAKLSQIVPNPTGT-EIKFTEDFARLTMDVQP-MRSMLMPETAKLIGSLFS

SLFPARVAAILN-AKMANS-RKMGELLHFLDEPSVVYNTY---KFQKGCVRDY-----SFIHEFIFDNDYVAEEE-
DIF-- LLDGLFNWSDRRKS-EKFVEFMRALDYQTNKVVTC--DIF-- LLDGLFNWSDRRKS-EKFVEFMRALDYQTNKVVTC---TFSGGVMRS--- - -GFLAEFFLDNDCEASEG -

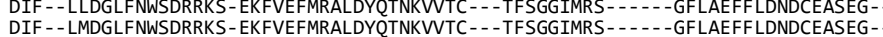

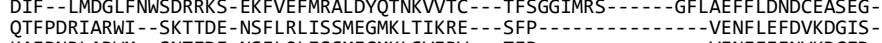
KAFPNRLARWM--SNTIDE-NSFLQLISSMEGMKLLSVERV---TFP-RSFPKRVRNHF --CKGYKE-SDFEQMLINSNGFQVKFKRC---EYPKNHL--SCSYAS-RKLEKFILEAEPFYLKVHRT ELFPERVDNAL --SSSYAS-RKLEKFILEAEPFYLRVRRT----.--SICGDRIRLNL--SSIYND-LSVENLIIDSKPYRVEFKRK---VRP----- - - - NVNDSWLDDVDQEGLE DIAGSLFTRLFN-RPEYDA-RCLEKFIRSCETTEIHVERR-YMEGIRRGA--GALGDSITYLID-KEQYKV-SSMEKF ILRCSSARITITRT -FRDYQPRRT----KLGELGTSWTGEEDEE DVMPHFIAASFF-AETEFH-KNFRDLNYNLATKGFEVLAIPMR-KALPDMLQRLF - - -PMKRHREVRQKFLVESPISASWDL KALPDMLQRLF - - PMKRHREVFQKFLVELSPISFSWDLR-- -ADLCP SLLPLFFRKML --GSFGR-DNIMKQIAMAEPFSVSIETC--R-1 DCLPDALHSAMF-RSHFKA-KQNLDLLMNMKTLSIVVETE---DFYPYSK--DLLPPSIHSALF-RKDFKA-RENLMVLIDLKPLTITLPTA---DYHMRSI----.-SSLPNSIARIF ---PQWKK-KNTFEFLFSLGTLVVDVERK ---VCFEHVLEEWGFEVVITDENAYLDPLSIFAIN SKMPNVVARMF - - -PQWKK-KNTFEFLFSLGTLVVEIERR---VCFEHILEEWGFEVVITDENAYLDPLSVFALN MALPNSIASCF -- DRWHG-LNIFHFLFTLDTIRVKVETK---V--KLFPMTIQRLS- - SDFKD-KNFFEVLFNCENLRVKIKTR- -SNLLPRYMORFF - - NSFKG YSLGKFIEETEPFSFTLRCS -- TYSRFG-EVLPRYMQRFF -- KSFKG-YSLGKFIEEIEPFSFTVKCR ---TYSRFS---

RKLPKPLAQRF - - KTVAE-VSLDDF IS IMEPFSFROELC - - D -

TFFPKYVAARF - - -DVARI-RSLDEFVEHMCPYTFKIKAV - - V- V

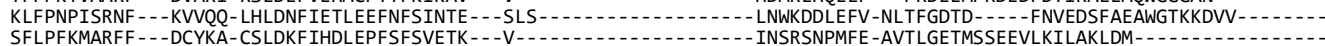

445

444

444

444

\section{- -DPKKEYSAHALRVNI- - - - KDRTPNPYGPMKARTPS -DPKKEYSAHALRVNI-- -DPKKEFSAHALRVSI - - - - - - RDRVPAPYGPMRAKTNS -DAKMDTSYSVATS--- -DPQPDTSYSVKVS-.-.-. RIDNRREEQYLYNKEALFNKN- - RIDSRGEGQYLYNKKTLFCEK -SGEADTSYSVSKSTLVC-}

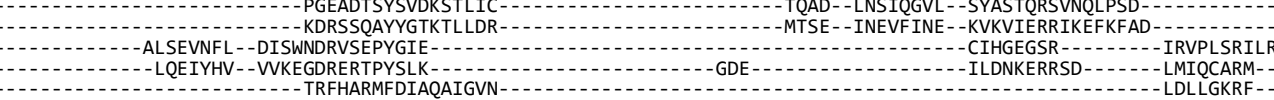

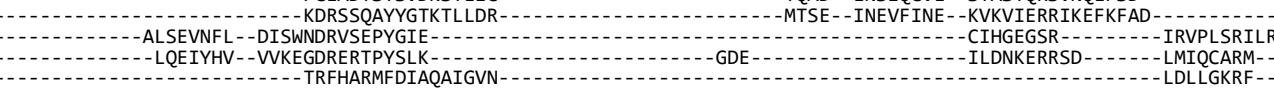
-
-1 -FNYNKRIFAIAEAIGVK -PKVKKKGLAKLI - - - PGREVDSHNYPREIYSDL - - LSSTSIWRSYDD -SEKKMIRDPLVKMIE - - - - - MGRESQGYSVVPTSYQQ - - - - AAVCIFSMDEN

-ERFNRGFCKSPVKI--- -ALKAKEGAK--- - - - LNVLSNPTF --VVLHREYITKV- - - - -EFSSKAKK - - - - - -VNVLKERLTG VVLHREYITKIVLHREYITKV---- - ELSSKEKK

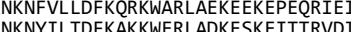
RIRELIDRRRCS

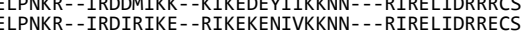
TQAD- - LNSIQGVL- -SYASTQRSINRLSSD-

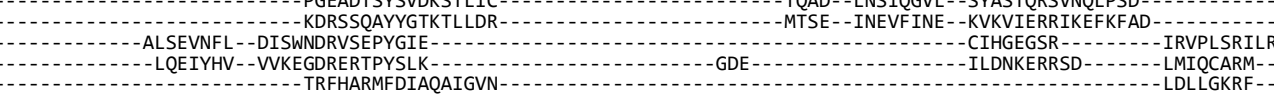

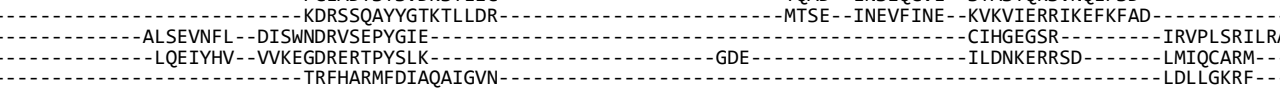
- ALSEVNFL--DISWNDRVSEPYGIETLLDRGDDFDCSKGKYASONEES-1 - - RDRRNIVNGFTPSFYSY--_ -FLNSCDYLQRDNIDKIIRS-NDEQMEFKGVQEDLMNS - - KSRGAFLFGSNRGNYHI-ERIRLPFWNLNDYDL--- - KRKRVNAYNILSYRFEEERKI -KSDDKMSKSDFYRI - -KIYDERTDITKGAS -- - FILKSNRS -

-ESAQKGPNKMLQIEWYG

GDRRTVVIKSLDGLIK-- - FSRSKNLYKMHIIFLLN-FSWIPKNYDALIKPSLLRCA-

ESYPDLIFNAHTLVFASSHPSITLMLVKSFINMWV - - - - GKTNDRYYOSLVALRQ - -ALNQKGAKL- - - - - FMLHNESY -

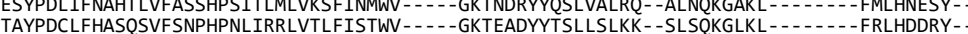
HKFXYGKVASL - - - LERQSQPYSGLVNGVKQPLLECPTECILKGFTTAIYKDLIDVYAKDVISCWVAVDVEHRLRNNTNYCVDIGAFKKLLLEPNHV- NITTVHHSPLVSKFESYGAQFHSILSVKSISAL TRIAKIVLSLYDPCV - DYPVIDAQKVFQLLRVISREWSFSFSMVQYCERGDYTEFMQASKLI-- 
AgVT
PVT:YP

PVT:YP 002019748.1

PVT : ADX 41471.1
PVT:AFU55321.1

PVT: AXK90539.1

PrVT:YP 009051684.1

PrVT: AHM̄M92766.1

ZoVT:QBS17025.1

ZOVT:QBS17031.

FiTA: :EDA2804.120

TrTA:OED42832.

GVA:NP 619662.1

ACVB: $: \bar{P}-004935358.1$

ASGV:NP- 044335.1

YaVA:YP-009268859.1
RAVA:YP 009553496.1

ACLSV:NP 040551.1

GPGV:YP
CtChV - 1 -Y YP -0097132978.2
CChV

CtChV-2:YP-009103996.

DiVA:YP 006905850.1

CLBV: NP 624333.1

CLBV:AFA43536.1
AVCaV:YP 008997790.1

CPrV:YP $-\overline{0} 09505632.1$
WVA:YP $009357235.1^{1}$

WVA: QEĀ69426.1

CRMaV:YP_007761581.1

CTLaV:YY-009046478.1

GCLV:YP_004936159.1

APV1:YP-

AgVT
PVT: YP 002019748.1

PVT: ADX X141471.

PVT : AFU55321.1
PVT : AXK90539.1

PrVT:YP_O09051684.1

PrVT:AHM92766.1

ZOVT:QBS17025.1

ChVT: QNG41875.1

FiTA:QED42804.

TrTA:QED42832.1

GVA:NP 619662.1

ASGV:NP-044335.1

YaVA:YY-009268859.1

ACLSV:NP -0405511.1

CtChV-1.YP -

CtChV-1:YP_009103999

DiVA:YP 006905850.1

CLBV:NP 629333.1

CLBV:AFĀ43536.1

AVCaV:YP 008997790.1

CPrV:YP $-\overline{0} 0950505632.1$

WVA:YP $\bar{\theta} 09357235.1$

WVA:QEAC99426.1
CRMaV:YP 007761581.1

CRMaV:YP_067761581.1

CTLaV:YP_-009046478.1
PhIVB:YP 001552317.1

GCLV:YP $\overline{0} 04936159.1$

ASVV:ND-604464.1
APV1:YP 099094347.1

AgVT
PVT:YP_002019748.1

PVT: AD̄̄ 41471.1

PVT : AFU55321.1

PrVT:YP_009051684.1

PrVT:AHM̄92766.1
ZoVT:OBS17025.1

ZOVT: QBS17031:

ChVT:QNG41875.

FiTA:QED42804.1

TrTA: QED42832.1

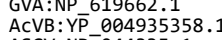

YAVA:YP 044355.1

YaVA:YP-009268859.1

ACLSV:N $\overline{\mathrm{P}}$ O405551.1

ACLV: YP 004732978.2

CtChV-1:YP_O09103999.1
CtChV-2:YP 009103996.1

DiVA:YP 006905850.1

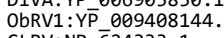

CLBV: NP $\overline{6} 24333.1$

CLBV: AFĀ A3536.1
AVCaV:YP 008997790.1

WVA:YP $\overline{0} 09357235.1$

WVA:QEĀG9426.1

CRMaV:YP 007761581.1

CTLaV:YP -009046478.1

PhIVB:YP 001552317.1

GCLV:YP -004936159.1

ASPV:NP_604464.1
APV1:YP_09094347.1

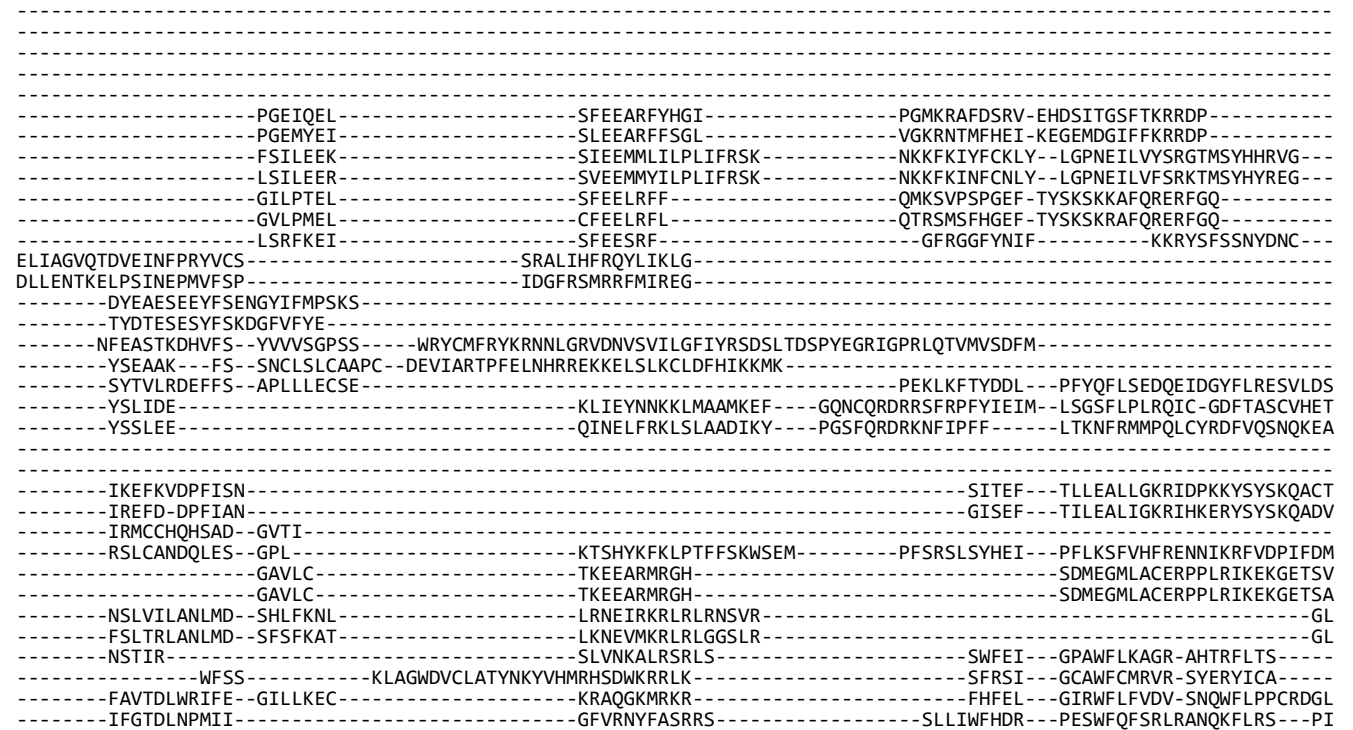

507
505
505
505
505
505
532
532
568
568
568
528
528
534
523
524
482
479
577
563
545
588
593
488
480
575
576
453
581
507
507
573
571
592
583
60
593
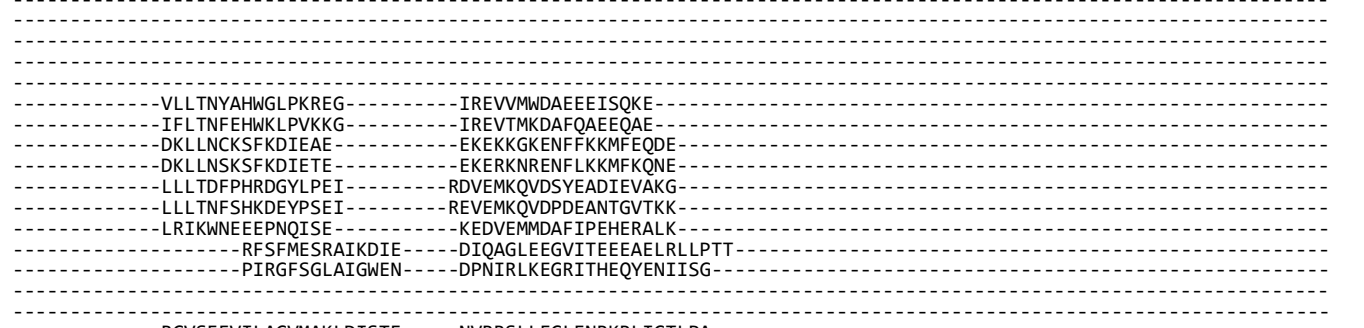

(1)

IKDKAFHYSEGFFDPILFASSRGTFLGHSEMTDL- - - - STSISLSKKGVKKAGRSRMIPVH

IKDKAFHYSEGFFDPILFASSRGTFLGHSEMTDL - - - - STSLNTLDKVIAENGEKSDVPIL

IEEVIDMLDMII---SPMPQIVTVEEIKDCLSVAEVESSIDPQEGNFQEKA-

ELHGPDSRHLMRSAHDCISYEANIKLF-GNNHMEKMRIE-

LSNYLTFLCA---EGLDGFNLEEHLERRLKAAGHDVS--DDEEEEL - TSAEQAGP--

(1)

作

IID-- EMNQLDLDIMNDGE---EAAVEILDVNSNLDGKQSEHHDEEFVTPTG - LKGGVE-1 -

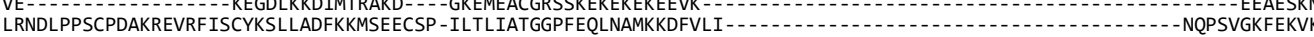

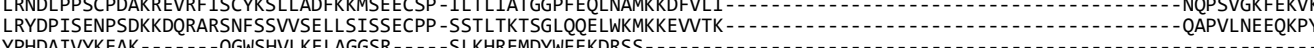
YPHDAIVYKEAK--- Q Q

YPDQAKSYKALD-

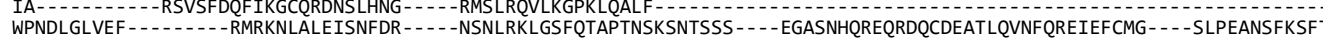

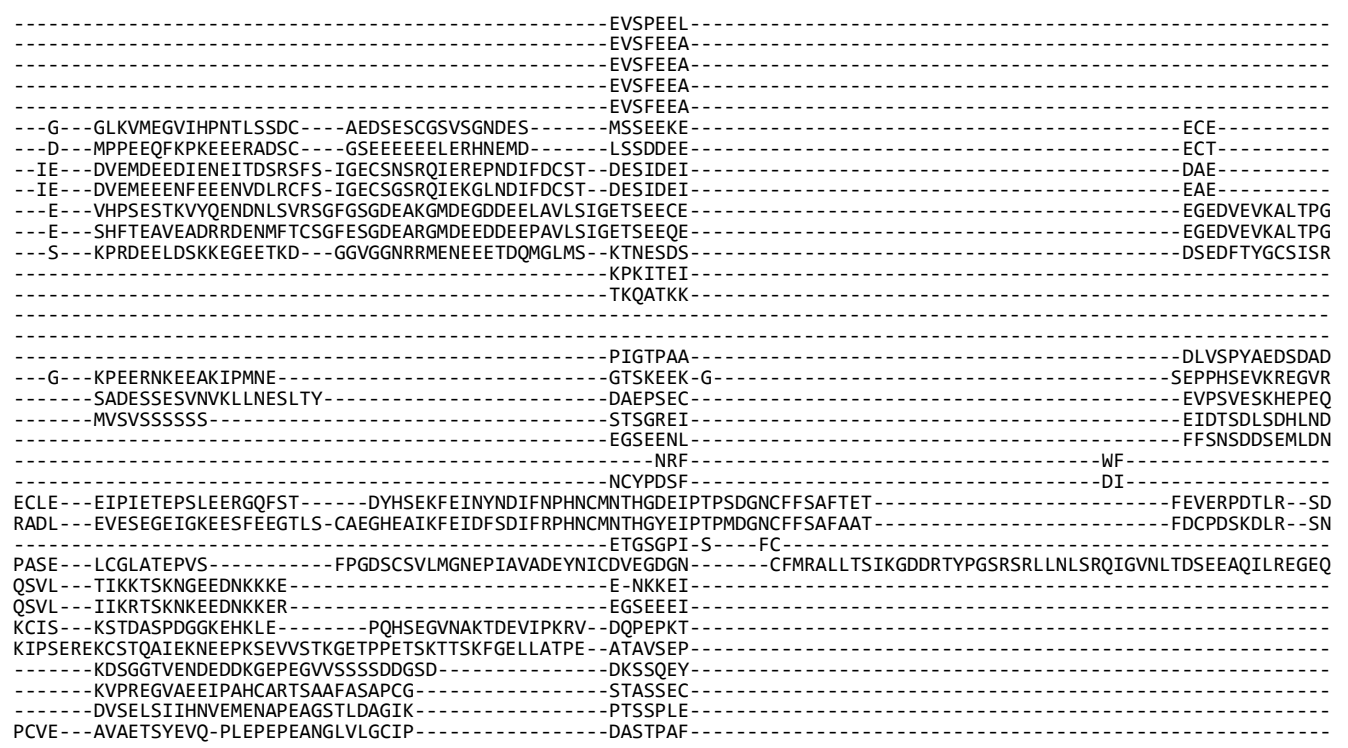


AgVT
PVT:
PFP

PVT:YP 002019748.1

PVT:ADX41471.1

PVT:AXK90539.1

PrVT:YP_ 009051684.1

PrVT: AHM̄92766.1

ZoVT:QBS17025.1

ChVT:QNG41875.1

FiTA:QED42804.1

GVA:NP 619662.1

ACV:YP 00493558.1

YaVA:YP_-009268859.

RAVA: YP ${ }^{-} 009553496.1$

GPGV:YP_ê04732978.

CtChV-1:YP-009103999.1
CtCCV-2:YP-0910396.1

DiVA:YP $000 \overline{6} 905850.1$

CLBV: NP 624333.1

CLBV:AFĀA3536.1

AVCaV:YP 008997790.1

CPrV:YP $009505632.1^{1}$

WVA:QEĀ 69426.1

CRMaV:YP_007761581.1

CTLaV:YY-009046478.1

GCLV:YP_ö 04936159.1

APV1:YP 099094347.1

AgVT
PVT:YP 0022019748.1

PVT: $:$ ADX $\bar{X} 41471.1$

PVT : AFU55321.1
PVT : AXK90539.1

PrVT:YP_O09051684.1

PrVT:AH92766.1

ZOVT:QBS17025.1

ChVT: QNG41875.1

FiTA:OED42804.1

TrTA: OED 42832.1

GVA:NP 619662.1

ACSBV:NP 044335.1

YaVA:YP-009268859.1

ACLSV:NP -04055111

CtChV-1:YP_009103999.1

DiVA:YP 006905850.1

CLBV: NP $\overline{6} 24333.1$

CLBV:AFĀ43536.1

AVCaV:YP 008997790.1

WVA:YP_O-09357235.1

WVA:QEĀ 69426.1

MaV:YP_007761581.1

CTLaV:YP- 009046478.1
PhIVB:YP 001552317.1

GCLV:YP 004936159 .

ASVV:ND-604464.1
APV1:YP 099094347.1

AgVT
PVT:YP_002019748.1

PVT: AD̄̄ 41471.1

PVT : AFU55321.1

PrVT:YP 009051684.1

PrVT: AHM̄M2766.1

ZoVT: QBS17025.1

ZOVT: QBS17031.1

ChVT:QNG41875.1

TrA:QEDD42832.

TRTA: QED42832.1

ACVB: $\bar{P}$ - 00493535358.1

Y

PaVA:Y_-009268859.1

ACLSV:NP 040551.1

GPGV:YP - CtChV 1 :YP 009103103999

CtChV-1:YP_-009103999.
CtChV-2:YP 009103996.1
DiVA.Y

DiVA:YP $00 \overline{6} 905850.1$
ObRV1:YP 009408144.1

CLBV:NP 624333.1

CLBV: AFĀA3536.1
AVCaV:YP 008997790.1

WTA:YP - 009535235.1

WVA: OEA 69426.1

CRMaV:YP_ 007761581.1

CTLaV:YP -009046478.1

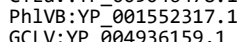

GCLV:YP $\bar{\theta} 04936159.1$
ASPV:

ASPV:NP_604464.1
APV1:YP_09094347.1

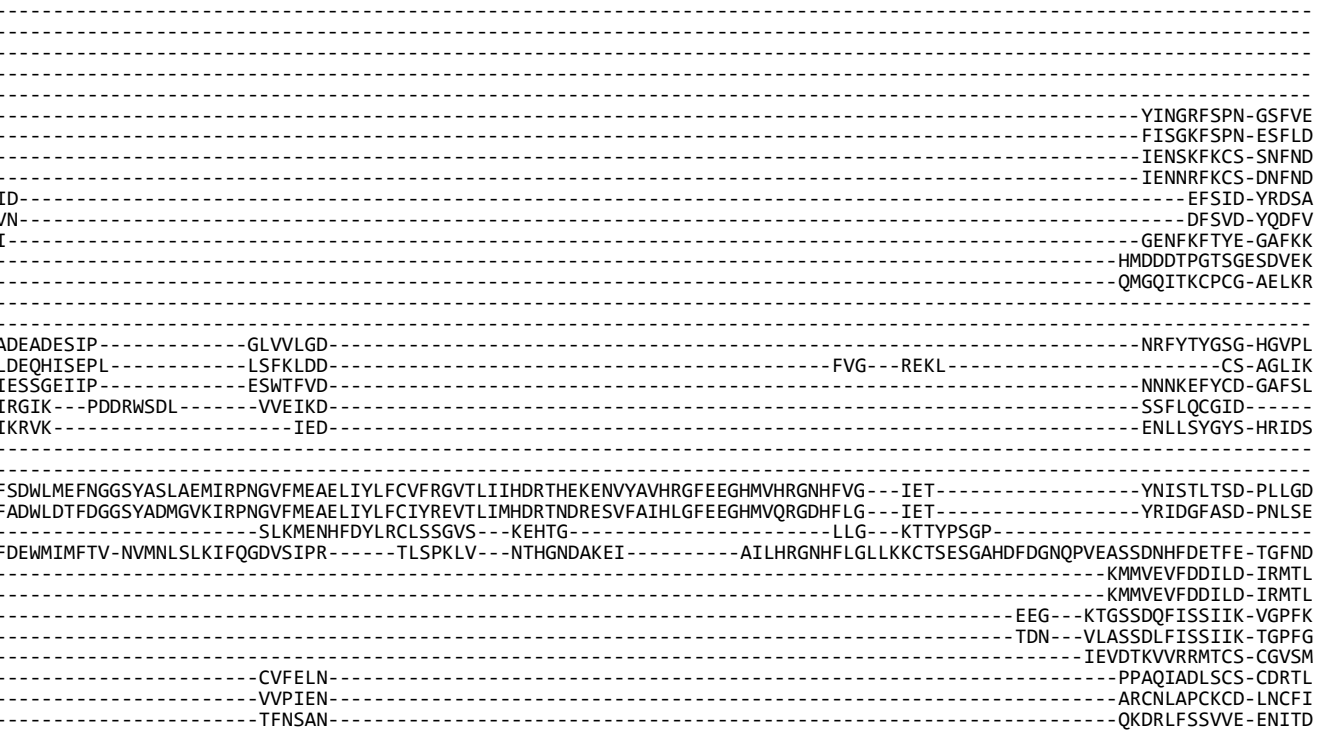

514

512
512
512
5

512
624

624

670

670
642
642

642

585

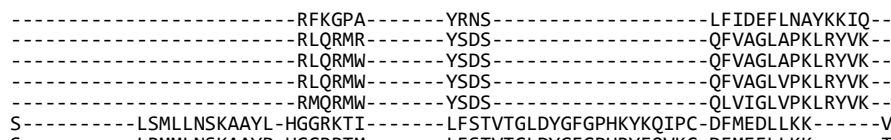

- IDGPRFVTD

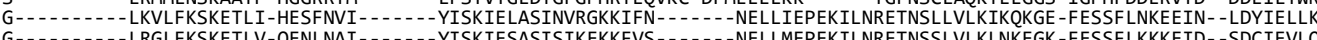
S-_-1

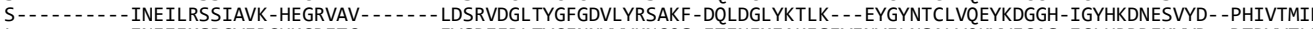
L----..--INEIFMSPGVFRGHKGRETC--.----FVSPIEDLTYGFNNVYYKNCQS-ETINEMIAKFSEYFNVELNSALVQKYVEGAS - IGLHRDDEKVYD- -DTPVVTLN F----KSVRSLCR--EEIYSEK-LKGREVA--..----FYSR-HSKEYKYNGGSHRS LGWDEALNELTQELG--LDDSYDHCLIQRYTAGGS - IGFHADDEPCYL-PGGSVVTVN T-.--KVEGGYRSLIPGWTDQ-LQGRKA--..---FYSR-HSEEYSYPGGSHKTRGWPAEIERMRNELG--LGEDFDHCLCQIYEKGAG-IPYHADDETCYK - -EPSVVTVN

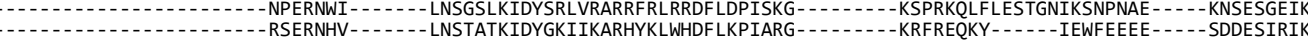

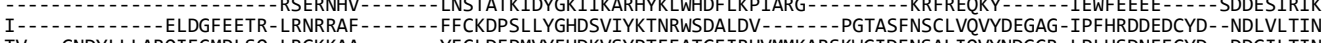
TV---GNDYLLLARQIECMPLSQ-LRGKKAA------YFCLDFPMVYFHDKVSYPTFEATGEIRHVMMKARSKWGIDFNSALIQVYNDGCR-LPLHSDNEECYD--DDGILTIN Le

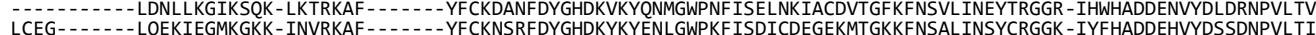

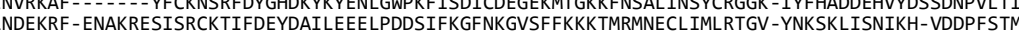
- LNDEKRF-ENAKRESISRCKTIFDEYDAILEEELPDDSIFKGFNKGVSFFKKKTMRMNECLIMLRTGV-YNKSKLISNIKH-VDDPFSTM IPCGFSEEITKFHFRPDHFNCAQ-FRGRKAA--.---FITK-VDADYGHNGMVYPHNSWVPSLEEIIQICG--QGDDFNCALINFYEANSS-LGFHRDNERVYN--DDPILTVC LPCGYSEELRNFHFKPEHFNCAQ-FRGRKGA- -...-- FLTK-VDADYGHNGMVYPHNAWVPSLDEIIRICD - -HGDDFNCALINFYGPNSS - LGFHRDNERVYN- -DDPILTVC

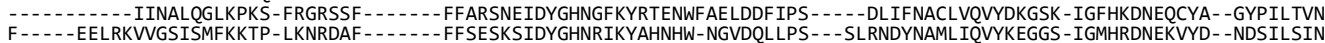
Fin T----NLLVKVL-ISKRMQLTR-FTSRDAY------YVVEDIRLDYGHNGIMYKKNLADERVRVFMEKVRETYKGKWNTMLIQRYKKGSS - INFHSDDEPMIM-RGSSVLTMN D.-.-NLLKKVL-ISKRMQLTR-FISRDAH-...-. D-..--HGVIEFIRS SFNDGHS-HNGRKAL-.....-FFSR-GGFAYGFNSVTYOSSGWPSAFEEI-.....-YGDRFNSCLVOKYFKSAK-LGLAKDDEDCYD-DEHEYMTVN E-..--IKTLLGSDMHAFKAKDR-LKGRVGG-...---WYSK-DGTPYLYTGGSHISOGWPDWLELWMOINK - -VPDKYNSCLYOTYEEGAS -VGYHADDEPIFK-KGEETHTCN

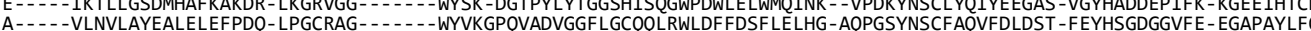

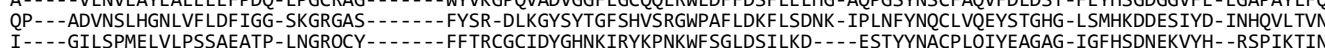

EFPSEESNEVEME -EFPSEESNEVEMEGFTSEESDEVEME

LEGEADF--TMFKKTGT-

VEGEADF--VMLKREGT

LKGYAIL--SIEENGTC-

LRGQASF--EIEGQ-

LKGRASF--EIEGQ---

LHGDATF-- EVKENQSGKI-

LFGEADF--KTKCTNE--

IEG-.-.-SAENDQPHEV

GKGSAAF--HISGDER- -

ENGTALF--EIKNV-

MIGEGMF--SVKMGKYE--

KHKRDRLN-

MNKKEKEM--..-..-

TFGEGRF--TIEFKDQV

TEGEGFF--SIEFKEQT
F-GLALF--EFDSG--

EHEEI-HDQQAADEEKPEEDSHNETEEEEAEIFSENSETLSE---FDDTKCDSFQIK-...-..--YDLL

DAFEL-RKGRTLKRGTKERDEDGANSEECEQLDSEDDVGSFD---YEETKADSYEID
DAFEL-RKRRTLXXXXKERDENGANSEECEQLDSEDDVGSFE---YEETKADSXEID

-DAFEL-RKRRTLXXXXKERDENGANSEECEQLDSEDDVGSFE---YEETKADSXEID
-DAFECEKENSEEEGSERDENGANSEECEQLDSEDDVGSFE---YEETKADSYEID DAFEYEQIEDAEKEGSEKEDEGEADLKEDERSSSEDDVGSFE---YEETKADSYEID -

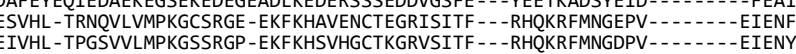

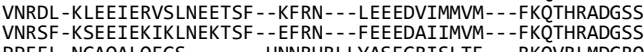
PPFEL-NGAQALQFGS----- -HNRRRRLLYASEGRISLTF-- RKQVRLMDGRQ - -

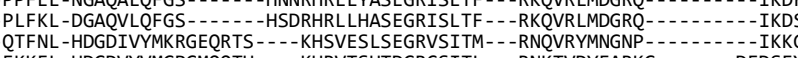
EKKEL-HDGDVYVMGPGMQQTH----KHRVTSHTDGRCSITL---RNKTVDYEARKG - - - - - DEDSEY

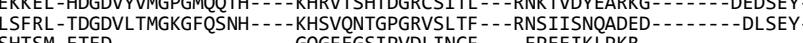
- GQGEESIPVDSTFF -

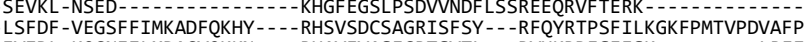

LVIDF-VEGSFFIMKADFQKHY----RHSVSDCSAGRISFSY---RFQYRTPSFILKGKFPMTVPDVARF

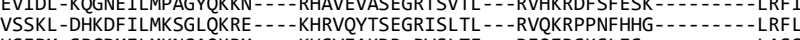

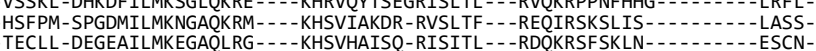
KVIKYYIGGVEYEMPSSQVSEL----EEITEITPINRLSSSNMILDFMKTGDTDDHVSKSRSTD TSFLM-TAGSFFLMPKGFQKKAASFLM-TAGSFFLMPRGFQRKAEQKTKEQTNLSICESK

RHSVSN-EMSRVSSTF---RKHVRRLNGSP

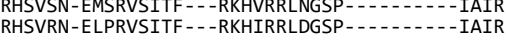

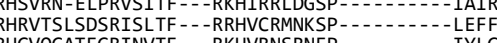
VEGSATF--RIVEKENEKKKKKKKENHPCPDEFLSL-NNFCVFKMKEGFQEL RHGVQGATEGRINVTF---RKHVRNSRNEP--

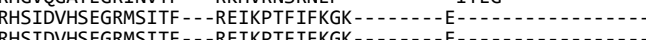

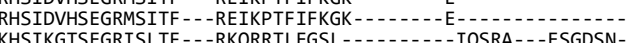

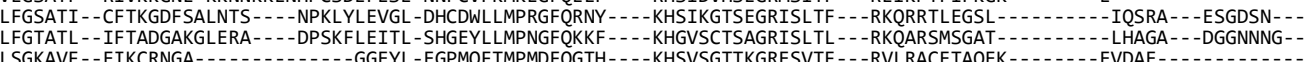
LSGKAVF--ETKCRNGA--.--

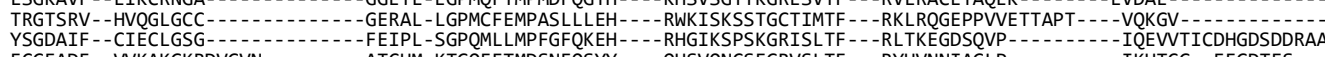

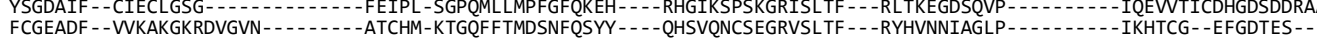



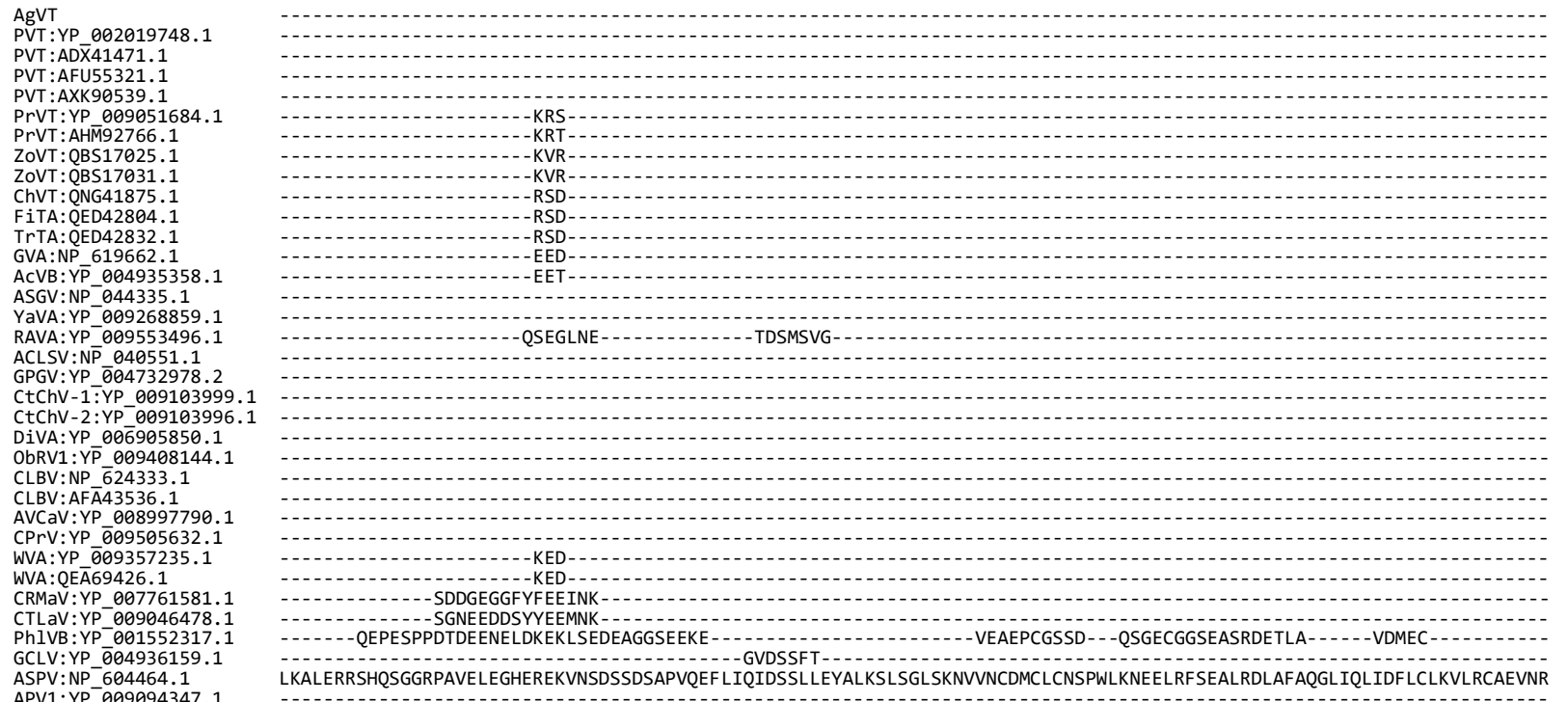

$\mathrm{AgVT}$
$\mathrm{PVT}: \mathrm{YP}$

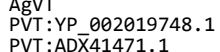

PVT:AFU55321.1

PVT :AXK90539.1

PrVT:YP 009051684.

PrVT: AHM92766.

ZOVT:QBS17025.1

ChVT: QNG41875.1

FiTA:QED42804.1

TrTA:QED42832.1

GVA $:$ NP 619662.1
$A C Y B: Y \bar{P}$
004935358

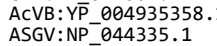

YaVA:YP- 009268859.1

ACLSV:NP -0405511.1

CtChV - - YP 00918392

CtChV-1:YP_-009103999.1
CtChV-2:YP 009103996.1

DivA:YP $006 \overline{6} 905850.1$

ObRV1: $\overline{\mathrm{P}} \overline{\mathrm{P}} 0099408144.1$

CLBV:AFĀ43536.1

AVCaV:YP 008997790.1
CPrV:YP 009505632.1

CPrV:YP $\overline{0} 09505632.1$
WVA:YP 009357235.1

WVA:QEĀ 69426.1

CRMaV:YP 007761581.1

CTLaV:YP- 009046478.1
PhIVB:YP 00152317.1

GCLV:YP -004936159.1

ASPV: NP 604464.1
APV1:YP- 009094347.1

AgVT
PVT:YP_002019748.1

PVT : ADX̄ 41471.1

PVT: AFU55321.1

PVT:AXK90539.1
PrVT:YP 00951684.1

PrVT: AHMM92766.1

ZOVT: OBS177025.
ZOVT OBS17031:

ChVT: QNG41875.

FiTA:QED42804.

TrTA:QED42832.

GVA:NP_619662.1
ACVB:YP_O04935358.1

YSVV:NP-

PaVA:YP-009268859.1

ACLSV:N $\bar{P}$ P40551.1

GPGV:YP 004732978.2

CtChV-1'YYP- 009103999.1
CtChV-2:YP 009103996.1

DiVA:YP_ 006905850.1

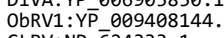

CLBV:NP $\overline{6} 24333.1$

CLBV: AFĀ43536.1
AVCaV:YP_008997790

AVCaV:YP_ 080997790.1

WVA:YP_009357235.1

WVA:QEAEG9426.1

CRMaV:YP_O07761581.1

CTLaV:YP- 009046478.1

PhIVB:YP -001552317.

GCLV:YP_- 04936159

ASPV:NP-604464.1
APV1:YP-009094347.1
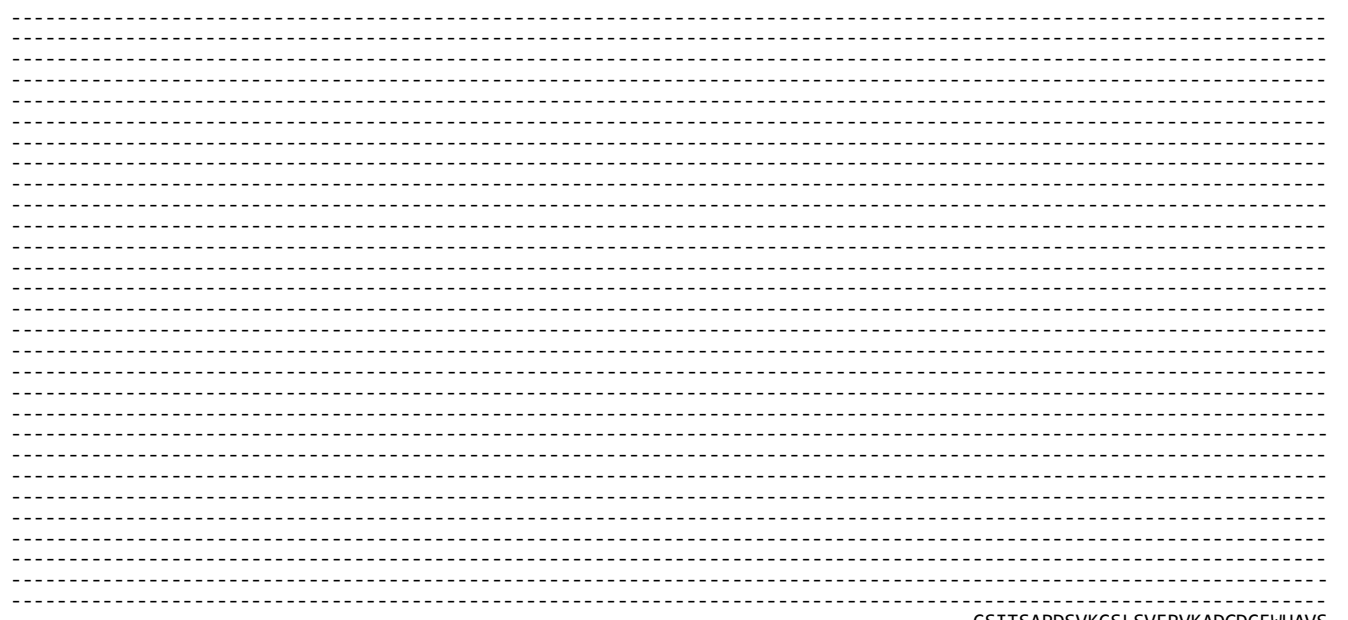

CSITSAPDSVKCSLSVFPVADGDCFWHAVS CSISSAPDSAKCSLSVFPVKADGDCFWHAVS

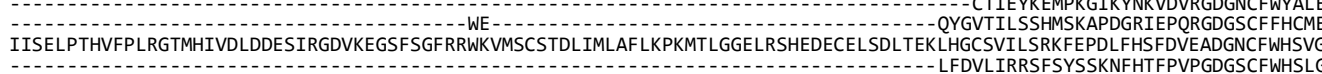

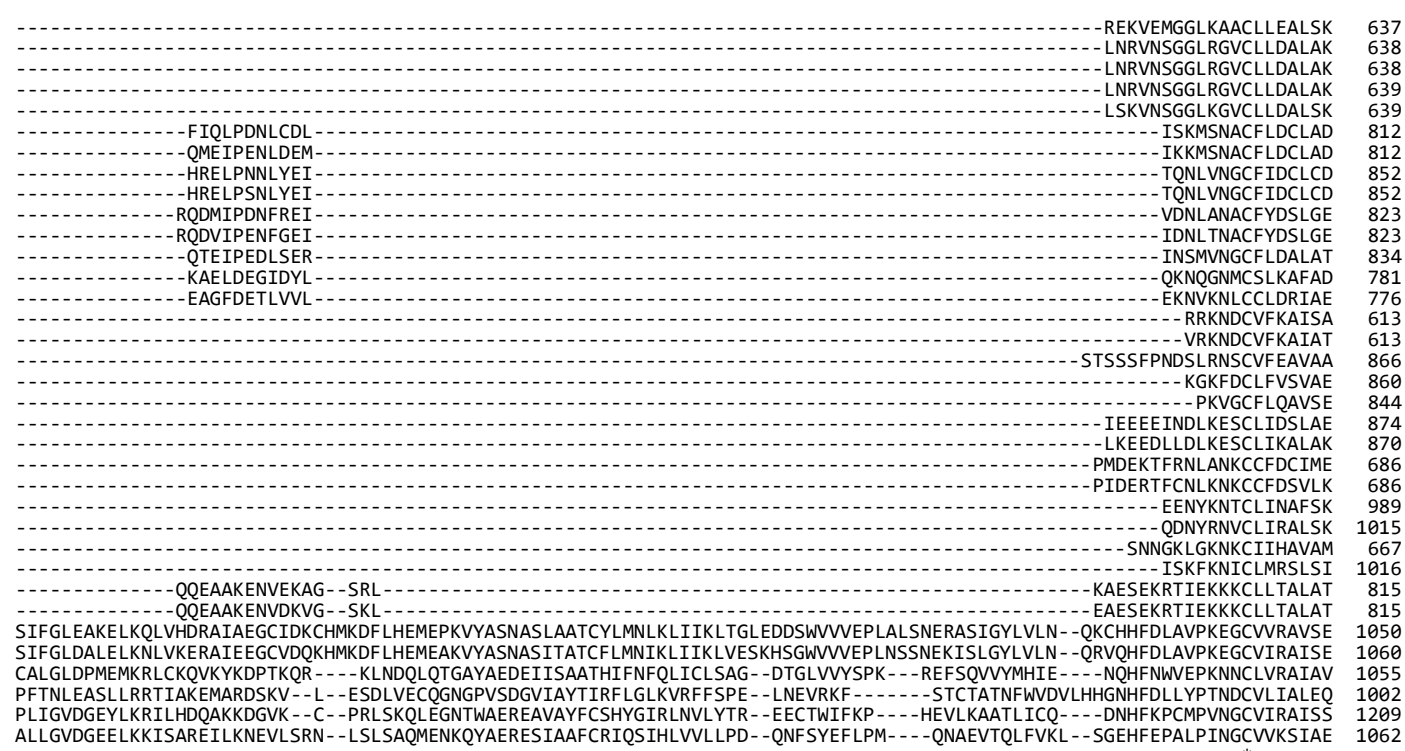


$\mathrm{AgVT}$
$\mathrm{PVT}: Y \mathrm{Y}$

PVT:YP 002019748.1

PVT:AFU55321.1

PVT: AXK90539.1

PrVT:YP 009051684.1

PrVT: AHM̄92766.1

ZoVT:QBS17025.1

ChVT: QNG41875.1

FiTA:QED42804.

GVA:NP 619662.1

ACVB: $Y \bar{P}-004935358.1$

ASGV:NP- 044335.1

YaVA:YP- 009268859.1
RAVA:YP 00953496.1

ACLSV:NP 040551.1

GPGV:YP_-004732978.2

CtChV-1:YY-009103999.1
CtChV-2:YP-0910396.1

DiVA:YP $000 \overline{6} 905850.1$

CLBV:NP 624333.1

CLBV:AFA A 43536.1

AVCaV:YP 008997790.1

CPrV:YP $009505632.1^{1}$

WVA:QEĀ 69426.1

CRMaV:YP_007761581.1

CTLaV:YP_-009046478.1
PhIVB:YP_001552317.1

GCLV:YP_ö 04936159.1

APV1:YP_009094347.1

AgVT
PVT:YP 002019748.1

PVT:ADX̄ 41471.1
PVT:AFU55321.1

PVT :AFU55321.1
PVT: AXK90539.1

PrVT:YP_009051684.1

PrVT: AHM92766.

ZOVT:QBS17025.1

ChVT: QNG41875.1

FiTA:QED42804.1

TrTA:QED42832.1
GVA:NP 61962.

ACVB: $\overline{\text { P }}$ - 004935358.1

ASGV:NP- 044335.1

YaVA:YP-
RAVA:YP
00926853595.1

ACLSV:ND 0404551.1

CtChV-1:YP_009103999.

DiVA:YP 006905850.1

ObRV1:YP_099408144.1

CLBV: NP $-\overline{6} 24333.1$

AVCaV:YP 008997790.1
CPrV:YP 009505632.1

WV:YP

WVA:QEĀ 69426.1

CRMaV:YP_007761581.1

CTLaV:YP_009046478.1
PhIVB:YP 091552317.1

GCLV:YP 004936159.1

ASV:ND-604464.1
APV1:YP- 099094347.1

AgVT
PVT:YP_Q02019748.1

PVT: AD̄̄ 41471.1

PVT: AFU55321.1

PrVT:YP 009051684.1

PrVT: AHM̄M2766.1

ZoVT:QBS17025. 1

ChVT: ONG41875.

FiTA:QED42804.

TrTA: QED42832.1

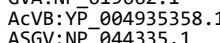

Y YVA:YY - 009268859

RAVA:YP - 00955349.1

ACLSV:NP 040551.1

GPGV:YP - CtChV 1 099103999

CtChV-1:YP-Y009103999.

DiVA:YP $00 \overline{6} 905850.1$
ObRV1:YP 009408144.1

CLBV:NP 624333.1

CLBV: AFĀ43536.1
AVCaV:YP 008997790.1

WTA:YP -009535235 .

WVA:QEĀG9426.1

CRMaV:YP 007761581.1

CTLaV:YP-009046478.1

PhIVB:YP-001552317.1

GCLV:YP $\bar{\theta} 04936159.1$

APV1:YP_009094347.1
VMRVKIEMVLAIL-CGRD--QTWADWFLKDIGAELADVEKALSDLDLPAIIHTE--DGVHKVD--GQG-----FKEVELWLHDNHVFT ITGTKREITLSIL-LGRD - -GTWADWFLKDKGATFDDVFKAVSDLDLNCTICTK - EGSFNAH--VNR----NYKHNFLYLFDEHVSLITGTKREITLXXL-LGRD--GTWADWFLKDKGATFDDVFKAVSDLDLNCTICTK-EGSFNAH--VNR----NYKHNFLYLFDEHVLLITGTKREITLSIL-LGRD--GTWADWFLKDKGATFDDVFKAVSDLDLNCTICTK--EGSFNAH--VNR----NYKHNFLYLDDEHVSL
ITGTRRETTSIL-LGRD--GTWADWFLKDKGATFDDVFKAVSDLDINCTICTK--EGSFNAH--VNKK---NYKHSFLYLFDEHVSL HLAMNRNGVFNLL-FDQD--RSVLTNVVEDKGFTLAEVIDHLTNLDIPGRIVSS--GEVINYL--EKG FLKMSRCQT ISIM-YSYD--DAVLDLIVNDKGF TVAEMINIL IKMDIPGVIFDG--KKNIRYL--EHG-
FLKMSRCQTISVM-YSYD--DAILDLIVNDKGFTVAEMINILIKMDIPGVIFDG--SKNIRYL-- EHG HLSLTRTQVISTL-IALD--GSWISKVINDSLVELSEVISALTALDIPGSINSK--GVRIDYL--REGHLSLTRAQT ISTL-IALD- -GSWISKVINDSMVELSEVISTLTALDIPGSINSK--GVRIDYL--KEG
HLKMSRESVVSFL-ISMD--DKWLQKIINDVQTSTEEMINTLVSLGIPGKIMNN- - GLIMNIP--GEG-

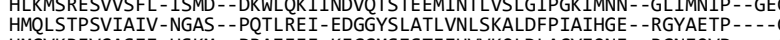

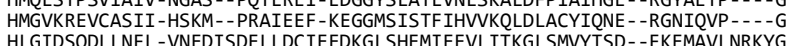
HLGIDSQDLLNFL-VNEDISDELLDCIEEDKGLSHEMIEEVLITKGLSMVYTSD--FKEMAVLNRKYG

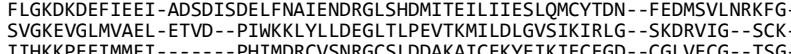

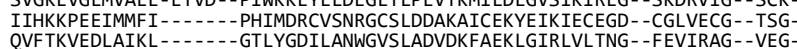

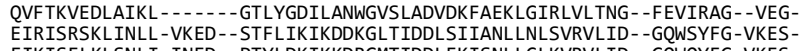

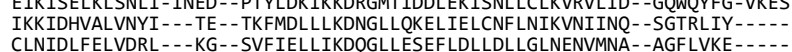

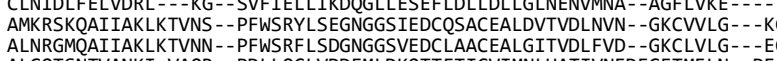

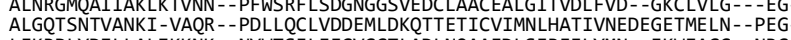

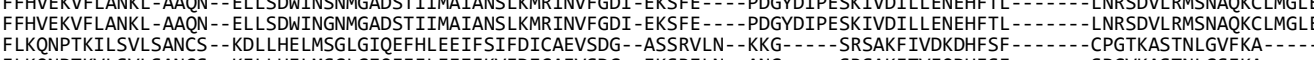

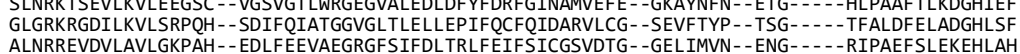
TLNQTEAKILSVIGRPNN--RLILEGLVEGEGLNIEDLEAAF SVFGICARVSTE- -RGVFTLN- -KEG CMNRSAVFNLL-FDOD--KSTITNVLEDGKATFDDVFAVVSDLDINCTICTK--EGSFNAH--VNK-

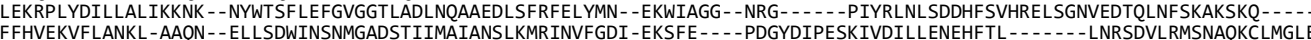

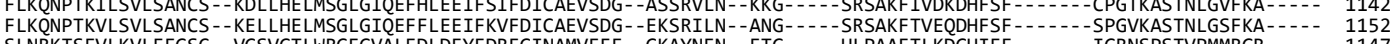

EKPLSFAQMGKFSRF-ERPKVMLFEQVRHQK ERPKIMLFEQVRHQK NVQHQVLYNSKDIRVE - ESLMYDGTGTLNID-EQTEAGTSLGEFTFK-ERTEAGTSLGEFTFKENGFEQEGVMKMSF FEGVTSKGGRE -KFRELKVSATGEHMSA- -1 LRGTIYCTIRNNHCEL-1 - VDKACFKKLLKDYEH--LSIGRMLLRGNHFSV----ASVRRSSMDSLANSSKEIK

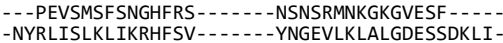
-NYRLISLKLIKRHFSV- - NFRGVSLRLSGSHFDA- - YNGEVLKLALGDESSDKLI法 VKTESISDWLLDDNK IPTLSRRKFSPIVSD - - - 1301 -NLHANYELKSGHIMY - - - - - LKKASSSQFAPTNPI - -

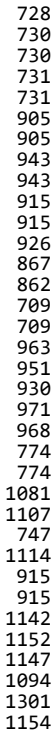

728
730
730
731
731
905
905
905
943
943
915
915
926
867
862
709
709
963
951
930
971
968
774
774
1081
107
747
114
915
915
142
152
147
1094
301
154
ERPKLMMFEKKKHQV

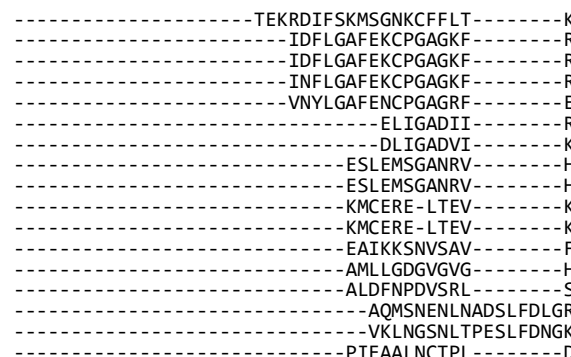

KYPFEIERADALIKSMKATYTGVVMSKFQKTPMLNGAP RYEALAERGSLLASALKDNLTGVISSKFNWDPKCEFVD RYEALAERGSLLASALKDNLTGVISSKFNWDPKCEFVD
-

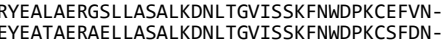
EYEATAERAELLASALKDNLTGVISSKFNWDPKCSFDN
KPNFSVERARKLVKSMMEGLTGVVLNRFKYAFNELLPH MPSYSVERARVCMKSLQEGATGIVLTKFKFEFNKILPN HPSYSVERARICMKSLQEGATGIVLTKFKFEFNKILPNKAEYSAERARKLIKSFQEGATGKILNRFKVGFNKVTMK AAEYSAERARKLIKSFQEGATGKILNRFKVGFNKVTMK FPKFNTERARRLIKSMQEGATGILLNRFKHAFNEISAK

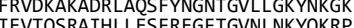

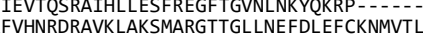

VKLNGSNLTPESLFDNGKVKHLKERAQKLAKSLNRGTSGILKEINSELASNLVEL

-SDG--VLDHVTFNFHKRLK--LVEPDLTNA -

-PKEFARKVSDE--MSNDANPQI IQNLNE-VYGVFLNQT KLSDSGFYNSF - (1) DIKVDSSRAGKLLKSLMDGMTGIVSHNSTHEGWRMIKGINSTSEM-RSFMNMVRGQIEEPKSDLFDK FKLDPKRAQRLLKSLLDGSTGVHCNSSLKEGWKMIPNAKSSEFVTKNYIN-----SSDI -WK FRINFERAALLIHSFLRGSTGVVTSSGFNNGVDYFSGRKRNIDP-ESF---1 - IKNVDYKRSKKLYDSLSKGTSGVFFNMIKKKNDESEKKKDKNRVI--EMMNFF-----FEDEI-NE QFAANFEFARILANSFLNMTTGICLGKALDNGEKYFLHI

EKGNVNVLEGFDAMLSGD-1-1,

KKSNLRVMDGLDEMLQSE-
DIPNCRSKKGIDICMSPDLANSNCAANYE--VTCQNLQVI

DRMEVNIKVRGNESGGNNRRQKLVNNVIEEILNFPSSSEV DRMEVNIKVQGNEFGSHNRRQKLVNNVIEEILDFPSSSEV -PSGCPMIAIEKY-DEFLRSSANVV PSGQTIPIEQY -ETFLRGNANVI - - VEASPHGRLVLNCAGTGV \begin{tabular}{l} 
LNRVSNSA- -MRFLAINGAEV \\
ONFNGQGP - -EIFLRGIVSEV \\
\hline
\end{tabular} Q QYQADHERAIKLMNSFLAGTTGAVLNELVFKGSRFFTFMD KFLAKKENALILMKSFLSMSTGICLSEHVHNGKDFMKLSKFLAKKENALILMKSFLSMSTGICLSEHVHNGKDFMKLS-

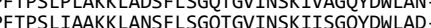

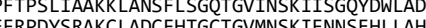

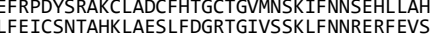
DYRPSIDRASTLLDSFEIGATGVLCQGIKEAQKDLASK

-DLTVGCLFGFAGSGKSRELTVKLRCYFNR IEKEILVVAGFAGSGKTRGICQIVKSMFNN

IEKEILVVAGFAGSGKTRGICQIVKSMFNN VKKEILVVSGFAGSGKTRGICQVVRAMFNK NENVMCIAGFAGSGKSRALQGVCASVLNK GNKVMCIAGFAGSGKSRALQGVCSSVLNK HSNKTFCISGFAGSGKSRGIQDLCCGILNS
HSNKTFCISGFAGSGKSRGIQDLCCGILNS FARTFVSMGFAGSGKSRSVQQMILGPGNT -FARTFVSMGGAGSGKSRGIQQMILGPGNT HTGEIEEPKEVLTAFGFAGSGKSHWCOTIIKHCSVE GTDIDNTLIDVYGLFGFAGSGKSYYPQTLLRCCNMK NYLPDHFESDLGMRLGFAGSGKTFKVLOWTKYTPTI-VYFKADLKMSFFSLMGFAGSGKTFKVLQWIKYTPTI VQELNFMKVKIYGIFGFAGSGKSHAIQNLIQTEFKG GSALWNAKINISGIFGFAGSGKSHGIQRLLNEKFSG -

EMTKGDAVVKGAVILGFAGCGKSRPVQMALDSMDSP KLIGEEFLIKGAAI IGFAGCGKSRPVQMALSNMDSP

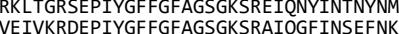
KDRVKQIGIDVTMVCGFAGSGKSRKLQSWLHSRKKG NERKSDFVEELSFVPGFAGSGKSLGLLNEVKRISREIHLAKEKKGMGKGSGKGHEKKERNRGNLKS SKREGDIISDLIVVSGFGGSGKSRSLOELIKEKKRG SKREGDIISDLIVVSGFGGSGKSRSLOELIKEKKRG TNKLCFERRVGATVGTFGSGKSHNVIELIRHNLG TNKLCFDERKIGAIVGTFGSGKSHNVIELLRHNLGY NINDRPNTVQINSVLGTFGSGKSSLFRRFFDANPGK - -STSLLPRTLNVICGVFGCGKSTLLCKALEKGLGV DRKLNDNSRNLGVVLGTFGSGKSSLFKRFIVKNPSR

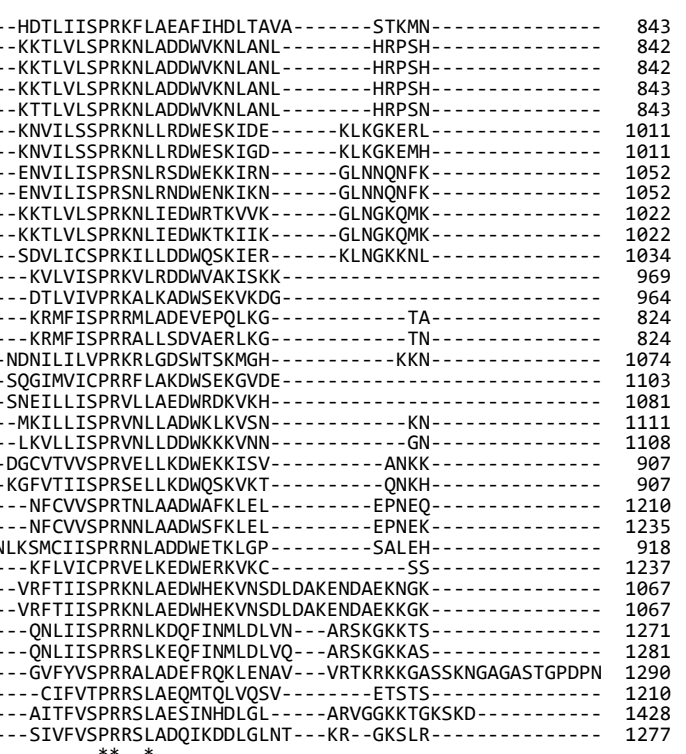

843 


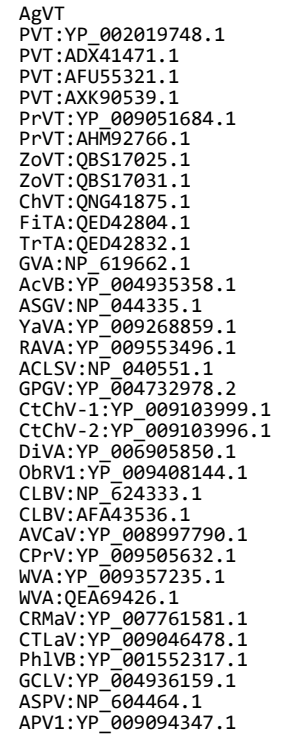

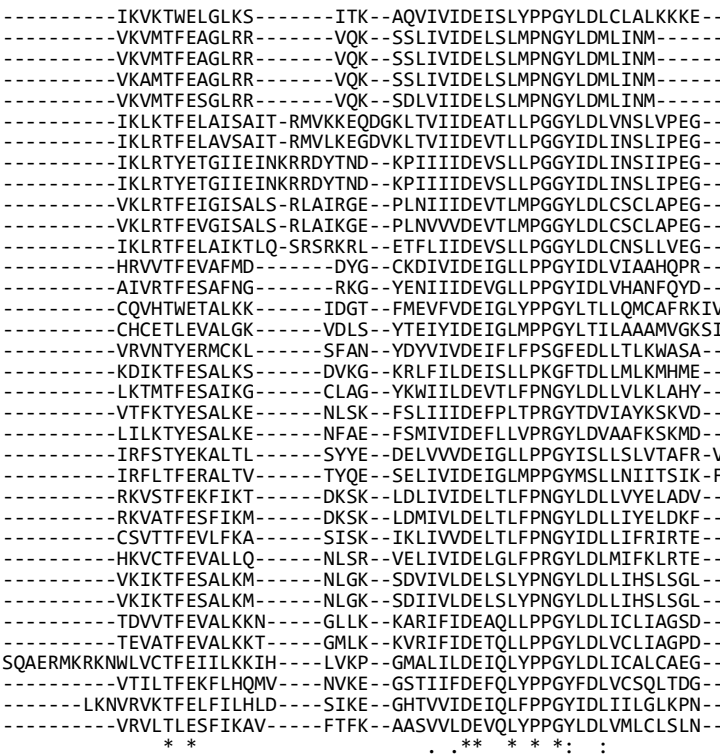

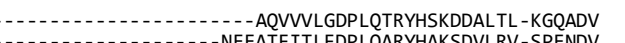
NEEATFITLFDPLQARYHAKSDVLRV-SPENDV -NEEATFITLFDPLQARYHAKSDVLRV-SPENDV -NEEATFITLFDPLQARYHAKSDVLRV-SPENDV
-NREATFITLFDPLQARYHSKSDILRV-SPENDV 913
- -VNLGPV
1088 --STIILLFDPLQSHYYSKMDVS---ANLGPV 1088 -STMVLIFDPLQSSYYSPKAVH---HSLPDI 1128 $\begin{array}{ll}- \text {-SIIVVIGDPCQAGYYSKDDVG---RNLGKA } & 1097\end{array}$ --SIIVIGDPCQAGYYSRDDSG---RNLGKA 1097 $\begin{array}{ll}- \text { SVIMLLGDPLQSTYHSKRDNVVLEASOEDV } & 1040\end{array}$ . \begin{tabular}{lr} 
& 1035 \\
\hline IVKGSENF - LKGKLLELSKTCLNIRCGDPLQLRYYSAEDTNLL-DKTHDI & 917
\end{tabular} 917
SIKSFSDKK--KFEAFKEMLPKLPRFNCLGDPLQCRYYCETDNALL -DKVDEI \begin{tabular}{ll} 
GILKKSTIVCIGDPLQAGYFCPKDDNYL-SREGEI & 1180 \\
\hline
\end{tabular} NEINLKHITLVGDPLQANYFNERDCNLL-GSVKMV 1158 NLTCRLEKKVTKLLLIGDPLQASYYSESDDDLL-AQGGEL 1193 \begin{tabular}{ll} 
& \\
-VNKISHNIRLSKRNYSKYVENQSSRLVLLGDHLQGRYYNESDFRSL-SQPDEI & 11902 \\
\hline
\end{tabular} $\begin{array}{lll}- & \end{array}$ -SPDCKLILIFDPLQARYDSAQDRAIL-GSEHDV 993 $\begin{array}{ll}\text {-KNFKGKVMLLFDPLQARYHSDSDERFL-HEIHEC } & 1313 \\ \text {-NVNMPRLVVIGCPFQARYHSKLDEHIL-TFDHEI } & 1143\end{array}$ NVNMPRLVVIGCPFQARYHSKLDEHIL-TFDHEI 1143 SSILVMGDPAQSSYDSAEDRMMFAGDKGCL 1345 $\begin{array}{ll}\text { - ASILVMGDPAQSSYDSADDRMAFIGDRGCL } & 1355 \\ \text { VHIVIGGDPIOSDYDNEKDRNWLSNLPPCI } & 1375\end{array}$ \begin{tabular}{ll} 
- ISLHLLGDPCQSDYDNAKDRGVFEGLLPDH & 1285 \\
\hline
\end{tabular} CQIYLAGDPCQSDYDSAKDRALFDGLKGDI 1351

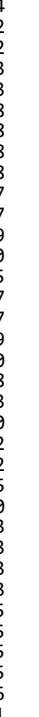

DRF----KIERYLLRSHRLSSE- - LSYM--

DRF---
DRI-- KVRKYLFFSKRMSSELDF--
DRI---KVPKYLFFSKRMSSELDF--DRI -- - KVPKYLFFSKRMSSELDFDRI----KVPKYLFFSKRLSSELDF-
LTPIFG-OEFRYRGYSYRFPKLFDLE LTPIFG-QEFRYRGYSYRFPKLFDLE
LTPIFG-QEYRYRGYTYRFPELFKVEFEPLYG-QSFDYKYYSYRFGDLFKIE
FEPLYG-OSFDYKYYSYRFGDLFKVE MDPLNN-CEFPYLFRTHRFPKLFDVE--10 MDPLNN-CEFPYLYRTHRFPKLFDIEFEPLYG-TSFKYLYQTYRFKNFQNFEFNRVRG--KLPYLCYSHRLPRNCKL FDRLMG--KKNYLYKTHRLPSNQKLDLMIKT-IKHKYLFQGYRFGQW--FQEL DFIRKSFKNFKYLFQGFRFGKW--FSEI
GRLIA--KEQPYSLSTRRNEGW-- IERI GRLIA--KEQPYSLSTRRNEGW--IERI
KRLFKG--GVNYKWYSYRINKF--IAKK DSVF---KDVKYQYQSYRIPAN--VAGR ETLEI--RKPKYLYYSHRLSSS--LGGIDFIMMN-EEILYLNYSHRLNKMHFYK VFLMEN-EEIIYLNYSHRMSRSHHYKDRLIGG-QNIEYIYSTHRMSRY - -FNRF DRLIGG-QDLRYIYSSHRMSKY--FNRF DLILGD-SEVDYMYQSKRFESEELFNLF
DRITSG-AKINYLFESWRLSKKFFGNFF DRIFKGNSAINYLAFSHRLGTG--FNCV DRIFKGNGAINYLAFSHRLGVG--FNCV DRLLE KKYYYSSESRFRNPMFLG DSVLEG-AEYKYVIKSRRFKNGNFOG-DSVLEG-AEYKYVIKSRRFKNGNFQG MRILSG-RSYKFNILSQRFRNPVFYG
FEVLSG-KKYKFNVSSRRFOSEMEVG
-FEFPCLSSEKLHELH-GKIYRQEEAL -FDVRCSSDQKKWELH-GKQYREPAAL FDVRCSSDQKKWELH-GKQYREPAAL -FDIKCSSDQKKWELH-GKQYREPAAL FDIGCSSDQKKWELH-GKQYREPAAL -GFE-FGKGEIDKNH-MRIFAQPQA - GLSMMGGMEISEHH-MKIFKQPEA -GLSMMGGMEISEHH-MKVFKQPEA GLQFLGKQEISEFH-MHEFGSPEA -NLVSMSAAALDENH-MKFYLQPEA FEIECMGAESEKRVVVNMPTRVDESKFS-- RKFFADIS VNIPTRDDESKHS - - -RKFFPDMS -

-

LAIETMNDF IGIDEQ-SSIYKDMPSAHH-FME FDVWDKNRHEPIDCH-GTFYSDLSSAKL-

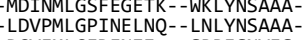
PGVEMLGEDENII ---SRRFSNVFS-FDVPCFNQADRTEEQRLWIFDDVYSIPS - ICSDRQEPCDVLLVESDLEKKA FDVPCFNQAETTKEQRLWILDDVYSITS -VCIDQGEPCDVLLVESDLEKKA

SVDLKGSDVQWLVASQNMKRKY--- - - - SHRGVPKTFGEVQGLTFNF-C 100

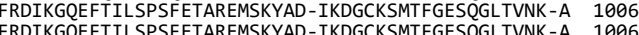
$\begin{array}{lll} & \end{array}$ $\begin{array}{lll}\text { PRDIKGOEYTILSPSFETAREMSKYAD-ERNGCKSMTFGESOGLTVNK-A } & 1007\end{array}$ -VREAIKRPVFLCPSDDKRSEL-------SNFGEAYTFGTSQGLTFDF-V 1176 -VKKVFSDPIFISPSEAKANEL-----SNFGEAYTFGTSQGLTFDF-V 1176 -VKKILNDPIFISPSEAKANEL----RRYGDSYTFGTSOGLTFDF-V 1217 -VLKKIEKPIFLCPSDAKRAEL- - - - - -SHYGDAYTFGTSOGLTFDF-V 1186 -VLKKIEKPIFLCPSDAKRVEL----SHYGDAYTFGTSQGLTFDF-V 1186 -VRAAIRRPIFLCPSEEKREEL----KRYGDAYTFGTAQGLTFDF-V 1198 GAEGDANYDLITTASRAME --

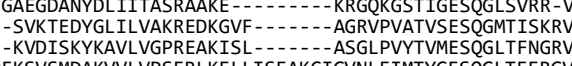
AGRVPVATVSESQGMTISKRV 作 $\begin{array}{ll} & \end{array}$

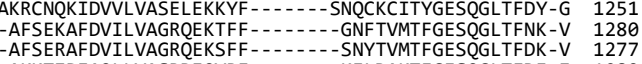
-KELDAKTFGESQGLTFDE-I 1089 -RDLGAKTFGESQGLTFDE-S 1089 SPIINVMTFGESQGLTFNH-V 1381 SPVINVMTFGESQGLTFNH-V 1406 $\begin{array}{ll}- \text { ASSIKTMTFGESQGLTVDH-A } & 1097 \\ - \text { AGKVNVLTFGEAOGLTVKH-S } & 1409\end{array}$ $\begin{array}{ll}- \text { AGKVNVLTFGEAQGLTVKH-S } & 1409 \\ - \text { SGLINVLTFGEAOGLTVNN-S } & 1240\end{array}$

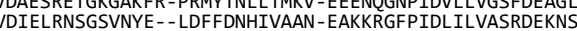

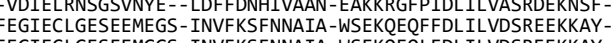
\begin{tabular}{ll} 
SGLINVLTFGEAQGLTVNN-S & 1240 \\
\hline
\end{tabular} -RLPCTFDSSRLTLE-KEEYAVFDSFKAFKADYLSPKIKTFLVSSFTEKTVVKANM---GRNVSIFTFGESTGMNFDY-V 144 -RLPCTFDQSRMTLE-KEEYAVFSSFKDFKNDYLSPKIKTFLVSSFTEKTVVKANM---GRNVLVYTFGKSTGMNFDY-V 1454 -RLPCEFGTQMEGQA-TEEHLLYSGLEH-LHVIPQEFSKVFLVSSFEEKKIVEAHFP--GSNPTVLTFGESTGLNFKY-G 1474 $\begin{array}{lll}\text {-RLPCEFGTQMEGQA - TEEHLLYSGLEH-LHVIPQEF SKVFLVSSFEEKKIVEAHFP--GSNPTVLTFGESTGLNFKY - G } & 1474 \\ \text {-RLPCAISNTNEDD--FEDFEILEGI ---EQVQEIDVECYLVSSF IEKQAVRALV---GLDKVVQTFGESTGLTYDC-V } & 1379\end{array}$

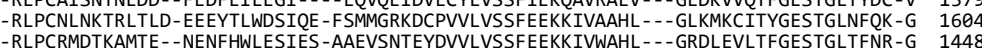

AgVT
PVT:YP 002019748.1

PVT: $A D \bar{X} 41471.1$

PVT: AFU55321.1
PVT :AXK90539.1

PrVT:YP 009051684.1

PrVT: AHM̄92766.1

ZoVT:QBS17025.1

ChVT:QNG41875.1

FiTA:QED42804.

TrTA:QED42832.1

ACVB:YP_ 004935358.1

YaVA:YP - 009268859

RAVA:YP 009553496.1

ACLSV:NP 040551.1

GPGV:YP_004732978.2

CtChV-1:YP_009103999.1
CtChV-2:YP-009103996.1

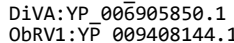

CLBV:NP 624333.1

CLBV:AFĀ43536.1
AVCaV:YP 008997790.1

CPrV:YP -009505632 .

WVA: $P$ - 69926.1

CRMaV:YP_007761581.1

CTLaV:YP_009046478.1

PhIVB:YP_001552317.1

GCLV:YP_-0 04936159.1

ASPV:NP_604464.1
APV1:YP_009094347.1
VVVICSDAHLVSNFAWMVALTRGRSGFCFLVDSVNDKEAVRINLQGRLIEKAMSKKKVTNTFLRAMAGVSLETAEFIED---VETFKTTESVEEKLEGDPWLKSLVPLLEYPEAF 1115 VIVVDQDLVATSVLHWIVALTRSRQGFVILVHKVFDMKTLIQPVQNSIIGLVLRGVKVQENIFINTAGKCLSEAEIVEE ---LETFKRTEEDEDLLEGDPWLKGQLFLCQSVELD 1118 VIVVDQDLVATSVLHWIVALTRSRQGFVILVHKVFDMKTLIQPVQNSIIGLVLRGIKVSREHLINTAGKCLSEAEMVEE --- - LETFKRTEEDEDLLEGDLWLKGQLFLCQSVELD 1119 VIVVDQDLIATSVLHWIVALTRSRQGFVILIHKVFDKTLIQPVQNSI IGLVLRGVKVSKEHLINTAGKCLADAEIIED --- LETFKRTEEDEDLLEGDPWLKGQLFLCEAVELD 1119 CISIDMDGSVTSDFHWMVALTRARRGFCFLTCASTSMRTFMDNNRAKLIGKVLKKEQISKKFWWNLGGRALEGARAVKKDEFSKLGKTREEFEESLEGDPWLKGMLNYLEGDDAN 1291 CISLDMDGSVTSDFHWMVALTRARKGFCFL VISVDMDGPLVSNAHWMVALTRARKGFAFVVCSSITLNDFKAKVKTKIIGKVLNKAVVSKDFMRASGGK IMDHANLIGD - - SKKGRTREEFEDTLENDPWIKTQLIFLENPELQ
VISIDMDGPLVSNAHWMVALTRARKGFAFVVCSSITLNDFKAKVKTKIIGKVLNRTIVSKDFMRASGGKIMDHANLIGD - - SKKGRTREEFEDTLENDPWIKTOLVFLESPELO IISIDMDGPVTDNCHWMVALTRAKRGFAFLNCPT IRRCDFLRQSEGKL IHKILKKQKVSMDFIKFMGGKIMRKARML - - - - -EAVGRTREEFEEACEGDPWMKAQLSFLEINIPE 129 VISIDMDGPVTDNCHWMVALTRAKRGFAFLNCPTIRRCDFLRQSEGKLIHKILKKQKVSMDFIRFMGGKVMKKAQML - - EAVGRTREEFEEACEGDPWMKAQLSFLEINIPE 1296 VISLDLDGPITSDAHWMVAL TRGRKGFAFLSCPTIKKKDF LDRTEGKLINKVLNGMKISLSYLRGLGGVMENVTFYDEN- -EKVGRTREEFEDILEYDPWLKAQINFLELNDV- 1310
LIYLDEHWAKKEDEDVMVALTRSRGETGIHVT-PALKKKL ITNAKSTLLKKVLKGETYRRSEIVAMVRKHIPETTVLFE - - ESRLAETVDYEARLAGDPYLKSLLALYDEIEME LIYLDEHWAKKEDEDVMVALTRSRGEIGIHVT -PALKKKLITNAKSTLLKKVLKGETYRSEIVAMVRKHIPETTVLFE---ESRLAETVDYEARLAGDPYLKSLLALYDEIEME
KLVIDRDWGLLNDKAVMVALTRARNTLSVEVD-KSMKEHLKVHAKSSILKMFLRGQMIKRELIMEMMGTDNGDVELIEK---ETRFADSDDMEDLSGDPYLKGLLRLYDDVEME
1224 LICLDEQILAGGPSVAITAITRATDGFDFVMR -GSSPNDLRRSANKGIWQFLMEQKEVPMERIVNL----LPGASFYEDSF----EVGNSSIQDKASNDLPMIMPPFINLAEEECDP 1112 VVMVSDETKKVSFGHLLVALTRSVRPPLIWTRRTFDL---VMRGNPLLDAIRERRIVKVSEM---1--VKRDMGFKVDF--EKIGRLE-FAEEKLHGDEHLLCIMDINESALPE 1344 VIVLSEEAKLCSDAHIMVAITRFRRGFCFALGSKGSKEDYMRSMKSGLLQRICSGVGASKEFILGSSSV ---NLILSEKDIAKGAGIDEMDREARLEGDVLLSMIYLGKRYHMV 1386 LISLSEESRLCSDNHIYVALTRFKKGFGFFQNFRGDLGTFKSNLGSKLLGRYINLRDNLKPFMMQMLDI ---NLDFMDD ---RNQVGAGIEMENKMSGDPWLKGLLDLQAVEEVE 1360

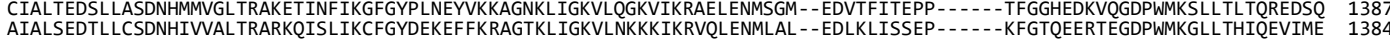
IIVLSPPAVNCSINMWNVAMTRARKGVHFALNGFDTVDF INRVKGTPVNAMILGSPFEIHRTPGGKDK ---EIKIIKV---CRLGMSNEDVEMKLMGDPFLKSIIPSLDEGLSI 1198 IIVLSPPSTNCSLFMWNVAMSRSIKGVHFALNGFDSIDDFLNRVKGTPVAAMILGMKFDIHAQPMSTPE---DCKIICS---DRLCLSSSDVENKLEGDPFLKSIIPSMDEGLCV 1198 CILLSESSAASNEFRWMVALTRARTRFSLCSTFLGGIEEFKVKRKESLITSILQGEKITFNRLNLML - - CILLSESSAASNEFRWMVALTRAKTRLSFCSTFLGGMDEFKIKRGESLVTSILEGKQITFERSNMMV--
AILLSENSALSDDHRWLVALTRARKKVTFLCLHLSGLNGFLSTMENRLVAAVINKGLVTKKRLSSMVRA CIVLSEYAEKQDDYRWVVALTRAKEKISFITSHRSGLTGFMSSMIGRPIHAFLTGLPFTSNRMNWMV LIVLSENSANSEEFRWVVALTRARRTLSFLVVHLDGIEGFMAETDGKMINALLRGEKVNVKEFSKKKG CVLLTQDSMLVDERRWVVALSRAKINISFINLSGLTLPEFCTQMMGGVVHKFFTSTATFNDLRELLPGD CVLLTQDSMLVDERRWVVALSRAK INMSFVNLSGLSLPEFCTQMVGGVVHKFFTGTATFNDLRSLLPGD TIIITNVAHTSEKRWVTALSRFSENICFVNLVNLSWSELARMYATRVLGRFLGKRAKLSDLLEHLPGV AVVVSEASKLASERRWITALTRARKRVTFITNLGCSKHLIAEIFSNRALGRFLSCTASIDNLRCLLPGE
AILVTYESALTSDRRWWTALSRFSHDIHFINGMGVTWDNAITHFVGKPLHKFFTKRACNDDIIDLLPGR -KCNLIRREK-ENGCRDEVDREERLEGDPFLKPFIFLGQRVEKD -KCNLIKQEK-KNGCSDEVDREERLEGDPFLKPFIFLGHRIQKS -KLNYVKFK - -GLAGKDEVDREDRLEGDLFLKGVIFLGQRCEIM
-NCELVECHR -ATGGRDEVDREDRLEGDPFLKPFVFLGQRINSE NLNFIEFNEIKNGGNDEQDRELRLEGDPWLKPFINLHQRENVE

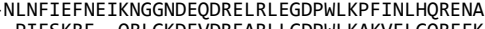
-PIFSKRF - -QRLGKDEVDREARLLGDPWLKAKVFLGQREEK I -AVFTDSYD-ENIGKDEGVREEKVQGDPWLKGMVDLFOIEDV -PNFVEELV-PTIGANLGVVEEKVSGDPWLKTMLFLGQVADVA
-PELIEGFQ-SOVGADEGVREAKLVGDPWLKTKIFLGQNPDFE -PELIEGFQ-SQVGADEGVREAKLVGDPWLKTKIFLGQNPDFE
-PELTNDFG-DKVGRSEGVMEAKLSGDPWLKTEIDLLQDEDQE

AILVTYESALTSDRRWTALSRFSHDIHFINGMGVTWDNAITHFVGKPLHKFFTKRACNDDIIDLLPGR
IILISHESTLTSERRWITALSRFRLNIIFVNLVGNCLEDACQVFHDRTLDRFLTKRATIANIVDQLPGL 


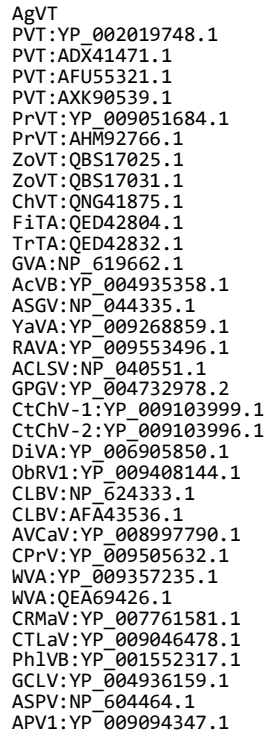

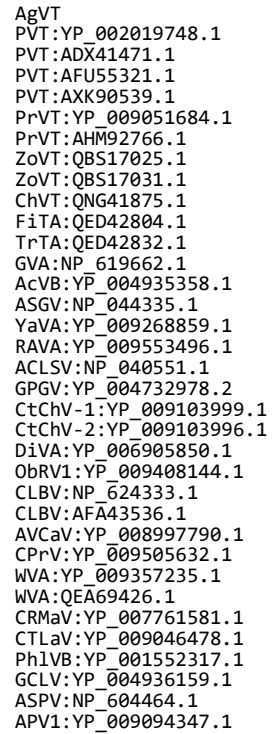

DIE-PREELKHDSPPKTHLCMASKAHT-SILMNEAKGREGREFRSIS EVT-PEEPLRHESPPRTHLPLPVEGLT-PLLMSNVKAREDREFITPS EVT-PEEPLRHESPPRTHLPLPVEGLT-PLLMSNVKAREDREFITPS EAV-PEEPLRHESPPRTHLPLPVEGKV-PLLMSDIKAREEREFVTPC DPE -PEEPIRKDSPPRTHLMIAPVEHQFAEEMHLLRAREFREFRNSN
DPE-PEEPVRKDSPPRTTHLMLAPVEHOFAEEMHLLKAREFREFRNSN DPE-PEEPVRKDSPPRTHLMLAPVEHQFAEEMHLLKAREFREFRNSN KEE-MNEMVFKSSPPRTHLMISSEGNAF INGPHLNRAREFREFKGRG
KEE-TNEMVFKSSPPRTHLMISNEGNAFINGPLNKAREFREFKGRG EPM-VEEPVRKDSPPRTHLLHGCESTAQIQGPGMNKAREHREFKSKD EPM-VEEPIRKDSPPRTHLLHGCESTAQIQGPGMNKAREYREFKSKD EVE-VEEPNRKESPPRTHLSIQPEGQAFVDGPSLLKSRESREFRGLG DIE-IEEPVTLE-PTKTHLALSTKMNELAP --FDLKAKEHREQHTEA EEE -VPDVSLPE-PQKTHLPISTKENELAP --SLLRAREHREARTPA EEV-VGDVIQPVEWFKCHVPVFDTDPTLAEIFDKVAAKEKREFQSVL EEV-VGEIVEAPEWFKCHVPVFDCDPMLAEMFDKIAAKERREFTSML EPL-GQVIKLTDDAIKCHIPVCSSQTL-GPELDNIQAREYREFKGKNDMF-FEDLNIIEPTGKVHLPLASRNDE - ---FEKIRARESRELKKLDEVE-LIEPDIVESKMKVHINITDKSYALMIINDQLRAKENREFKSKD-EQE -YHDIICESPVPKIHLPIESIQGHVSYVSSMLKERGEREFKGDG EQE-YHDIICSPVPKIHLPIESIQGHVSYVSSMLKERGEREFKGDG
HHE-YQDVFELPTPKIHLPIESIQSHVAFVSSMIRNREFREFIGDG EDE -VEEVKIREPTCQTHLYITEPNFGLCYNFDFIREKEQREYREDM - HDE -VGE IEVREPTCQTHLYITEPNFGLCYNFDF IREKEQREYREDM- EPE-IVEPVMAKEDMKTHFFVCQENFAQCYNFDNIRAKELREFRIGHEYE-IIEPEVIEPKGRVHLCISQENYALARNFDLIRAKEYREAKLMG EVI -VDEVF IKEDKEKTHLYLAEPNFSQALNFDLILDKEVREFRLGE
EVI -VDEVFVREDKEKTHLYLAEPNFSOALNFDLILDKEVREFRLGE EVI -VDEVFVREDKEKTHLYLAEPNFSQALNFDLILDKEVREFRLGE VEEPISVENLKDIKIKVHCPVGSMGATFAEVOSKLKVKEAREHRIDT- EEE-EOLEEMOTEWFKVHLPOAELESVRAKWVHKILAKEFREVRIGH DEI-DVDEALQIEPFKTHVARSNLEGVRALWHDKLLLKEHREKRMGY MEE -LAEEVKHEPWFKTHLPLFELESIRASWVHRIMNREYREVRCGS
-GWSEQFSDLDHKKGR-KFPDVSYAEAYETIYPKHTLSDDVTFWAAIQKRIVKSNPQKEAR 1219

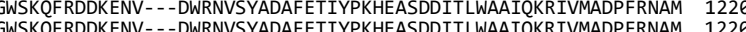
GWSKOFDDKNV---DWRNVSYADAFE TIYPKHEASDDITLWAAIQKRIVMADPFRNAM 1220 -GWSKQFRDDKENV---DWRNVSYADAFETIYPKHEASDDITLWAAIQKRIVLADPFRNAM 1221 $\begin{array}{lll}\text { LWSEQFDDCRKTRKV----IHNRAETFEQIYPSHKNSDTLTFWAAIKKRMKMSDPYSERR } & 1393 \\ \text { MWSDOFDDSRKTRKV- - - - IHNRAETFEOIYPSHKNSDTLTFWAAIKKRIKMSDPFSERR } & 1393\end{array}$ MWSDQFDDSRKTRKV--- IHNRAETFEQIYPSHKNSDTLTFWAAIKKRIKMSDPFSERR 1393 $\begin{array}{lll}\text { MWSEQFDDCRKERKF----KYNRAETFE TIYPNHNGTDSLTMWAAIKKRLKMSDPYTERR } & 1431 \\ - \text { MWSEQFDDCRKERKF----RYNRAETFETIYPNHNGTDSLTMWAAIKKRLKMSDPYTERR } & 1431\end{array}$ TWSSQFQDDNSSRN - - - - -LDNSAESFESIYPKHSNSDVLTMIAAIRKRLKFSDSNSERR 1397 TWSSQFQDDNPSRN---LDNSAESFESIYPKHSNSDVLTMVAAIRKRLKFSDPSSERR 1397 LWSEQFDDLKKGRRL----LNNEVERFENIYPRHNASDSLTFWAAIKKRLKFSNALTERR 1412 GRTEQIDEN- - - GYQGEVGDPMTHKALYLRHTSDDTATFMMSVKKRLRFRNYEANRR 1326 GTTEQIDEM- - GYKMEPENPMTHKALYLHHRNSDVATFFLSVKKRLRFMDREKNHR 1321 GLSNQFLDMEKN---GCKIDILPFARQNVFPHHQASDDVTFWAGVQKRIRKSNWRREKS 1213 GYSNQFLDMEKK ---GSNIDVYPMSRQSVFPHHQGSDDVTFWAGVRKRIKKSNWRREAT 1214 $\begin{array}{lll} & \end{array}$ DWSMOFEDCGVKIK --RVLNGNLCENFSAVYPVHOACDEMTFLAAIKKRLRFDNVANNYA 1490 SWSNQFKDNDQNLNL---ETSTGPVNFEAIFPRHQTFDDVTFWMAVKKRLSFSNPLVESE 1490 CMSEQFPDFWKTGE--PGHYLSQSERFQSIFPKHQNSDSLTFLAAVKKRLKFSSPSVERE 1302 LVTNOFCDSYDKVHINGKRETPGPLRFKAIYPKHSADDDMTFWMAVRKRLVFREEEENYQ 1596 RVTNQF IDNYEIVOHVOKKHTAGPLRFEAIYPRHCADDDVTFLMAVHKRLRFSNEMKERE 1313 LETNQFCHDYNRVGAQGSRHVASPLRFESIFPRHRSDDDLTFWMAVKKRLRFSEEFLERA 1624 $\begin{array}{lll}\text { EQTNQFTDNYNVNHWGGKRINAAPFRHKAIYPRHEMKDDLTFKMAVKKRLRFEEPAVNYQ } & 1458 \\ \text { EQTNOFTDNYNVNHWGGKRINTAPFRHKAIYPRHEMKDDLTFKMAVKKRLKFEEPAVNYO } & 1458\end{array}$ $\begin{array}{lll}\text { EQTNQFTDNYNVNHWGGKRINTAPFRHKAIYPRHEMKDDLTFKMAVKKRLKFEEPAVNYQ } & 1458 \\ & 1657\end{array}$ $\begin{array}{lll}\text { LVTEQFSEVHKGK -- -GKVLTAAPDNFEAIYPRHKAGDTATFVMAARKRLKFSFPARERQ } & 1657 \\ \text { IVTEQFAEVHKGR ---GKILTAAPDNFEAIYPRHKAGDTATFVMAARKRLKFSLPAKEKQ } & 1667\end{array}$ LVSEOFTDEHPREO--GKQLTNAAERFEAIYPRHKANDTVTFMMAVKKRLKFSRPATEKA 1688 $\begin{array}{llll} & \\ \text { LVSEQFTDMHSKNM--GKKLTNAAERFETIYPKHKGSDTVTFIMGARKRLRFSKPAVEAR } & 1593 \\ \end{array}$ -ETTTQFPDDHPSGA--KITLANAAERFEAIYPRHRGSDSVTFLMAVKKRLSFSQPSKESA 1662

KLERDMPIGK---EILNEVLKVYPLENIW- -VDLAEHEKNFFKRRVEKSKGL IOSHSNRSDPDWKLDHFFLFMKSOLCTKKEKRFCDAKAGQTLACFAHOLLCRFGVPFRVFE 1327 $\begin{array}{lll}1327 & 1329\end{array}$ KLXKVEPISA----EIFNEMNKILLLNPHV-SVDRDQVYKEFLRKRLNKSKKLIESHSERSSDDWPIDHFFLFMKSQLCTKFEKRFVDAKAGQTLACFSHKLLTRFGPAFREFE
KLOKVEPISA----EIFNEMNKILLLNPHV-NVDRDOVYKEFLRKRLNKSKKLIESHSERSSDDWPIDHFFLFMKSOLCTKFEKRFVDAKAGQTLACFSHKLLTRFGPAFREFE KLQKVEPISA----EIFNEMNKILLLNPHV-NVDRDQVYKEFRKRLNKSKKLIESHSERSSDDWPIDHFFLFMKSQLCTKFEKRFVDAKAGQTLACFSHKLLTRFGPAFREFE
KLOKVEAISA----EIFNEMNKILCLNPHV-SVDRDOVYKEFLKKRLNKSKKLIESHAERSSDDWPIDHFFLFMKSOLCTKFEKRFADAKAGOTLACFSHKLLTRFGPAFREFE KLQKVEAISA----EIFNEMNKILCLNPHV-SVDRDQVYKEFLKKRLNKSKKLIESHAERSSDDWPIDHFFLFMKSQLCTKFEKRFADAKAGQTLACFSHKLLTRFGPAFREFE 1330 KLERCMPIGE---NLCKIFIEEYGLKRGV-IVDVESTEREFLLKRVEKAKKMIEAHSERSDPDWLVNHFFLFMKTQLCTKFEKRFSDAKAGQTLACFSHQVLARFGVPIRIAE 1502 KLERLMSTGK----SLFEIFKKEYGLRRDV-RVNTDEIYADFIDRRLNKSKALISAHSERSDPDWICNHFFLFMKTQLCTKYEKRFSDAKAGQTLACFSHSVLTRFGVPIKEVE
KLERLMSTGK----SLFEIFRKEYGLRRDV-RINRDEIYADFIDRRLNKSKALISAHSERSDPDWVCNHFFLFMKTQLCTKYEKRFSDAKAGQTLACFSHSVLTRFGVPIKEVE RLERVKSVGI--- - SLFEIFCEYGIKRRR - FRDVEVIEOTFIEKRLNKSKKMIECHATRSDPDWSIKHFFLFMKSLLCTKFEKRHVDAKAGQTLACFSHKVLWRFGVIRAFE 1506 KLESLRGAGE- - - - NLFEIFKKTYEINNLF-KPDMELIEKTFIEKRLNKSKKMIEAHSERSDPDWKVNHFFLFMKSQLCTKYEKRFCEAKAGQTLACFSHRVLMRFGIKIREAE 1521 KYKTCHGIGH-----QMFSVFKDTYQKEIDSLPELERCEMEFMKKRIEKSTGLIEKHAGRSDPDWPSNYLKIFLKQQTCTKMEKRGVDAKAGQTIACFAHSVLCRFGPILRQTE 1436 RFNKVKGFGK----QLFKVLKETYNLRQPDKLPDLDRIEAEFARKRLNKSKNLIEKHSYRSDPDWPSHYLKIFLKQQVCTKMEKRGVDAKAGQTIACFCHAVLCKFGPLLRRTE
KFEEFESOGK----ELLQEFISMLPFEFKVNIKEIEDGEKSFLEKRKLKSEKMWANHSERSDIDWKLDHAFLFMKSOYCTKEGKMFTEAKAGQTLACFQHIVLFRFGPMLRAIE KFEEFESQGK----ELLQEFISMLPFEFKVNIKEIEDGEKSFLEKRKLKSEKMWANHSERSDIDWKLDHAFLFMKSQYCTKEGKMFTEAKAGQTLACFQHIVLFRFGPMLRAIE
KLESVQEDGK----ALLREFLKMLPKDFKVNTDDIDAGEKSFIEKRKOKTEKMWEAHSNRSDIDWNLDHVFLFMKSOYCTKEAKMFTEAKAGOTLACFQHIVLFRFGPMLRAIE KLESVQEDGK-----ALLREFLKMLPKDFKVNTDDIDAGEKSFIEKRKQKTEKMWEAHSNRSDIDWNLDHVFLFMKSQYCTKEAKMFTEAKAGQTLACFQHIVLFRFGPMLRAIE 1324 KFKAAESRGK----YLTKIFLKHVPIKCGRDQRLLDQCRQEFEETKLSKSAATIGAHSQRSDSDWPLDKIFLFMKSQLCTKFEKRFTEAKAGQTLACFPHKILVEFSPWCRYTE 1600 KFRGATAAGK-----ILLKNFLKF IPIPSETFPELLSEAKREFQEVKLKKSEGTIAGNSGRSDPDWSWDRVFLFMKSQQCTKFEKRFCEAKAGQTLACFSHEILCHFSPWCRYME 1571 KLNKAWIKGS----ILHKEFTRLIRVNSHFRPDLFEKALNDFEDVRMRKSEKLIMAHAGRSDPDWDIRNFLLFMKSQLCKKAEKAFCDAKAGQTIACFAHGVLFKFSAWCRYAE 1600 KLKSAFVKGS----ILYKEFRKIIRVQGDFRPDLFDKALSDFERVRVAKSKKLIEAHAGRSDPDWDVKKFLLFMKSQLCKKAEKAFSDAKAGQTIACFAHGVLFKFSAWARYAE 1597 RFEKVRHLGN-----EMLDIFLDKIKIDNKLNSEMMARSYNEYVLKKVSKTANTIASHSSRSEPDWKLNEIFLFMKTQLCTKFEKRFSDAKAGQTLACFSHIILNRFAAPTRYVE 1412 KFEKVRHLGS----EMFELLLEKIPLDNKNDDLMMQICVNEYIERKVSKPAGTIKSHSGRSDCDWKLNDVFLFIKTQLCTKYEKRFSDAKAGQTLACFSHVILNRFAAPARYIE 1412 RLSRAHLVGG----LLYTNFKKKMGLEFTFDQGLLESINAFEKKKLEKSCGTIKSHSIRSDIDWALNDVFLFMKSQLCTKYEKQFVDAKAGQTLACFQHLILVQFAPWCRYLE 1706 RLSRAHLVGG- - - - LLYRNFKNKLGLEFTFDQGLFEESVNAFEKKKLEKSCGTIKSHSIRSDVDWALNDVFLFMKSQLCTKYEKQFVDAKAGQTLACFQHLILVQFAPWCRYLE 1731 KLERAHGTGS----ILFHNLIQKLGLNFTWDNQLFECVNDFECKKLEKSKAVLANHSIRSDNDWSPNWVLFMKSQLCTKYEKQYVDAKAGQTLACFQHMILVTFAPYCRYME
KLKDSYSVGN----LLYONLKEKLSLSFSWDOGLLDECLNDFETKKLLKSKATLANHSIRSDIDWSMDKIFLFMKSOLCTKYEKOYVDAKAGOTLACFSHLVLAKFAPYCRYME

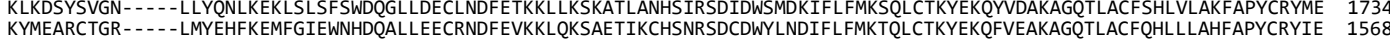
KYMEARCTGR----:LMYEHFKEMFGIEWNHDQALLECRNDFEVKKLQKSAETIKCHSNRSDCDWYLNDIFLFMKTQLCTKYEKQFVEAKAGQTLACFQHLLLAHFAPYCRYIE
KYMEARCTGR----LMYEHFKETFGIEWNHDQALEECRNDFEVKKLOKSAETIKCHSNRSDCDWYLNDIFLFMKTOLCTKYEKOFVEAKAGOTLACFOHLLLAHFAPYCRYIE KYMAAIPYGV----SMLQVFLKRIKLQSNFDHRLFEEARADFEEKKLOKSMATLENHSGRSDPDWSVEKALIFMKSOLCTKFDNRFRNAKAGOTLACFHHDVLCRLAPYIRYIE 1767 KFMSAIPYGD----TMLKVFLNKVRLKPNFDHRLFEEARNDFEEKKLQKSMATLENHSGRSDPDWEIEKALIFMKSQLCTKFDNRFRDAKAGQTLACFHHNVLCRLAPYIRYIE 1777 KLVEAMPYGK----FMLKEFLKKIPMNKSRDTKMMEQSKLEFEEKKLSKSAATIENHSGRSCRDWLIDIGLIFSKSQLCTKFDNRFRVAKAAQSIVCFQHEVLCRFAPYMRYIE 1798 KLMDASNFSE---FMLQEFLKHVPLKKPHNQAFMDASLADFEEKKTSKSAATIANHAGRSCRDWLIDTGLVFMKSQHCTKFDNRFRDAKAAQAIVCFQHAVLCRLAPFVRYIE 1703

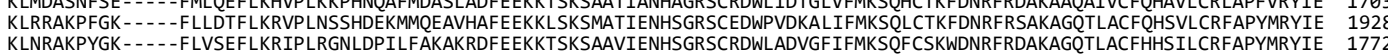

AgVT
PVT:YP_002019748.1 PVT:ADX̄41471.1

PVT:AFU55321.1

PrVT:YP 009051684.1

PrVT:YP_A $P$ PrVT :AHM2766.1

ZoVT:QBS17025.1

ChVT:QNG41875.

FiTA:QED42804.1

TrTA:QED42832.1

ACVB:YP_ 004935358.1

YaVA:YP - 009268859.1

RAVA:YP-009553

ACLSV:NP 040551.1

GPG:YP_- 04732978.2

CtChV-1:YP_009103999.1
CtChV-2:YP-009103996.1

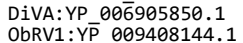

CLBV:NP-624333.1

CLBV:AFA43536.1
AVCaV:YP_008997790.1

CPrV:YP

WVA:YP

CRMaV:YP_007761581.1

CTLaV:YP_009046478.1

GCLV:YP_-004936159.1

ASPV:NP_604464.1
APV1:YP_009094347.1
EKVKKQLPKNVYIHTMKNFDQLNDWVKENV--DEHL-GTESDYEAFDRSQDSLILAFELHLLKHMGWDENLLQDYKVIKMYMGCRLGSLAIMRFTGEFGTFFFNTMANLAFTVLR 1439 KKFTANLPPSWYIHTMKNFDQLNNWVINYV--DQEE-GTESDYEAFDRSQDAIILGLEIECLKLFGWDQDLIDDYRKLKLRMGCRWGAIAIMRFTGEFGTFFFNTIANIAFTCLR
KKFTANLPPSWYIHTMKNFDQLNNWVINYV--DQEE-GTESDYEAFDRSQDAIILGLEIECLKLFGWDQDLIDDYRKLKLWMGCRLGAIAIMRFTGEFGTFFFNTIANIAFTCLR KKFTANLPPSWYIHTMKNFDOLNNWVINYV--DOEE-GTESDYEAFDRSODAIILGLEIECLKLFWDDDLIDDYRKLKLWMGCRLGAAIMRFTGEFGTFFFNTIANIAFTCLR 1442 KKFTANLPPSWYIHTMKNFDQLNNWVINFV--DQEE-GTESDYEAFDRSQDAIILGLEIKCLELFGWDQDLIDDYRKLKLWMGCRLGAIAIMRFTGEFGTFFFNTIANIAFTCLR 1442 KKLRAQLGENIYIHSGKQLDELNEWCMGYA---KGY-GTDSDYESFDRSQDALILAFELHLLRFLGWSVDQVEDYVTLKLRLGCRLGYLAIMRFTGEFGTFFLNTCCNMLFTCLR 14613 KRLRSQLNDNIYIHS EKMRFCLKDNIYIHSGKKLDELNNWCIKYA---TGY-GTDSDYESFDRSQDALILAFEIHLLEFFGWTKDLIYDYVSIKLRLGCRLGNLAIMRF TGEFGTFFLNTCCNMLFTCLR 1651

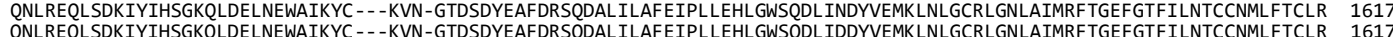
AKLRACLGENIYIHSGKKLEELNEWSKRYC - - -SDC-GTDSDYEAFDRSQDALILAFEIPLLRFLGWDESLVAEYIDIKLNLGCRLGYLAIMRFTGEFGTFFLNTCCNMLFTALR 1632 KALRELLPEKLMIYSQKKYMDLDKWAKTWV--ESMM-GTDSDYEAFDRSQDEKVLDLEVEVLRFFLWPEDLIREYEELKLMMGCALGDLAVMRFSGEFGTFFFNTVCNMVFSCMR 1548 KALRAQLGDNVIYSQLNYTDLDKWCKNFV--PSML -GTDSDYEAFDRSQDERILDFEMEVLKFFLWPEEVINEYKELKLMMGSSMGSLAVMRFSGEFGTFFFNTICNMAFTCLR 1543
SAFLRSCGDSYYIHSGKNFFCLDSFVTKNASVFDGF-SIESDYTAFDSSODHVILAFEMALLOYLGVSKEFOLDYLRLKLTLGCRLGSLAIMRFTGEFCTFLFNTFANMLFTOLK 1437 AAFLRACGESYYIHSGKNFFALDTFVTRNASFFDGE -SIESDYTAFDSSQDHTVLAFEIELLKHLGVSNEF IMDYKKIKLTLGCRLGSLAIMRF TGEFCTFLFNTFANMLFTNLK 1438 KLLLEMLPVNWYIHSGKNFNCLNDFVKTHL --KDGMECVENDYEAFDSSQDHSILAFEVKFLRLIGWPADVVDDYIHLKCTLGCKLGGLAIMRFTGEFCTFLFNSLSNMAFCCTQ 1679 KVLTANLPDNYYIHQRKNFSELEDFARRFS--NGSI-CVESDYTAFDVSQDHTILAFEVELLRHFGWDDRVLQSYIKMKCTLGCRLGGFAIMRFTGEFSTFLFNTLANMVFTFCR 1712 KVFSKYCPENFYIHQRKDFDKLAEFSRKYC - -KGGF -CIESDYVAFDVSQDHNVLAFEVQLMEHIRIPECVISDYIRMKTELGCKLGNFAIMRFTGEFCTFLFNTFCNMAFTFMR 1683 LKINEVMPEAFYVHSKKNFDELERWVGNF--IGPI-CVESDYEAFDASQDSTILAFECLILKDVGWHDLIEDYKTLKLELGCKLGMLAIMRFTGEFGTFFNTLANMAFTFCR 1712 KKISEGLGKNFYIHQKKNFDVLNDWVVANN - - FDSY-CLESDYEAFDSSQDCLILAFEYELLKYLGWDQSLLDDYLDLKFNLGCRLGNLAVMRFTGEFGTFLFNTLANMVFTFMT 1524 KKLSLCLPDNYYIHQKKNFDMLNAWVVRND--FSDE-CLESDYEAFDSSQDCLILAFEYELLKYMGWSQSLLDDYLDLKFNLGCRLGNLAVMRFTGEFGTFLFNTLANMVFTFMS 1524 TQIRNQLPEEIYIHSNKNFDDLNAWVKKFF --QRDI -CVESDYEAFDASQDEYILSFEIHLMKDAHFPQKIIDAYIDLKCKLGCKLGHFSIMRF TGEFCTFLFNTLANMAFTMCR
AOIRNOLPEEIYIHSNKNFDDLYRWVKNFF --OKDI-CVESDYEAFDVCODEYILSFEIHLMKDAHFPORVIDAYIDLKCKLGCKLGHFSIMRFTGEFCTFLFNTLANIAFTLCR AQIRNQLPEEIYIHSNKNFDDLYRWVKNFF --QKDI-CVESDYEAFDVCQDEYILSFEIHLMKDAHFPQRVIDAYIDLKCKLGCKLGHFSIMRFTGEFCTFLFNTLANIAFTLCR 1843
KQLRAQLPGEIYIHSNKNFNDLNEWVKKHA--GDDL-CVESDYEAFDASODOYILSFELFMMRHMHIPEOIIQAYIDLKVNLGCKLGHFAIMRFTGEFSTFLFNTLANMAFTMCR
1535 KQLRAQLPGEIYIHSNKNFNDLNEWVKKHA--GDDL-CVESDYEAFDASQDQYILSFELFMMRHMHIPEQIIQAYIDLKVNLGCKLGHFAIMRF TGEFSTFLFNTLANMAFTMCR
KMLRRNLKEEIYIHSNKNFNDLNDWVVKFF--EEGE-KVESDYEAFDASQDHYVLAFEVCVMEDMGLPNWFINDYIDLKCTLGCKLGHFAIMRFTGEFSTFLFNTLANMAFTFAR 1846 KQLTRMLPDEIYIHSSKNFDELNEWVIKHF - - QNDI-CVESDYEAFDASQDQYILAFEMALMKDAGMPDHILDDYLKLKCELGCKLGKFAIMRFTGEFCTFLFNTLANMAFTISR 168 - QNDI -CVESDYEAFDASQDQYILAFEMALMKDAGMPDHILDDYLKLKCELGCKLGKFAIMRF TGEFCTFLFNTLANMAFTISR 1680

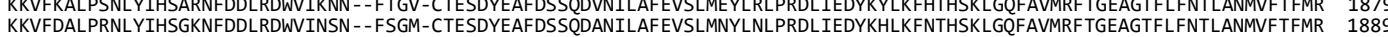
KKLHQALPGNFYIHSGKGLEELNEWVMRGR --FDGV-CTESDYEAFDASQDQYIMAFEIEMIKYLGLPADLISDYEFIKTHLGSKLGNFAIMRFSGEASTFLFNTMANMLFTFLR 1910 RKIAEVLPEKFYIHSGKGLEELNAWVTRGR --FEGV-CTESDYEAFDASQDQYILAFELHVMKYLGLPRDLIEDYKFIKMHLGSKLGNFAIMRFSGEASTFLFNTMANMLFTFLR 1815
SKVTEVLPKNLYIHSGKNIDDLAAWVTTSK --FNGV-CTESDYEAFDASODHFILAFELEVMKFLGLPSDLIADYTFIKTHLGSKLGSFAIMRFTGEASTFLFNTMANMLFTFLR YKLQAASPSNLYIHSGKNLEDLNEWVIRNK --FSGM-STESDYEAFDSSQDHFILSFEIEIMKHLQLPWDLIEDYVYIKTHLGSKLGNFAIMRFTGEASTFLFNTMANMLFTFLR 1884 
AgVT
PVT:
PFP

PVT:YP_002019748.1

PVT:ADX41471.1
PVT:AFU55321.1

PVT:AXK90539.1

PrVT:YP_009051684.1

PrVT: AHM 92766.1

ZoVT:QBS17025.1

ZOVT:QBS17031.1

FiTA:OOED42804.1

TrTA:QED42832.

GVA:NP 619662.1

ACVB:YP 004935358.1

ACGV:NP- 044335.1

YaVA:YP-009268859.1

ACLSV:NP 040551.1

GPGV:YP_004732978.2

CtChV-1:YP_009103999.1
CtChV-2:YP_009103996.1

DiVA:YP $00 \overline{6} 905850.1$

CLBV: NP 6243331

CLBV:AFÄ43536.1

AVCaV:YP_008997790.1

CPrV:YP 009505632.1
WVA:YP 009357235.1

WVA:QEĀ69426.1

CRMaV:YP_007761581.1

CTLaV:YP_009046478.1

GCLV:YP 004936159 i

GCLV:YP_004936159.1

APV1:YP- 009094347.1

AgVT
PVT:YP 002019748.1

PVT: ADX̄41471.

PVT:AFU55321.1
PVT:AXK90539.1

PVT:AXK90539.1
PrVT:YP_009051684.1

PrVT:YP 009051684.

ZoVT:QBS17025.1

ZoVT:QBS17025.1

ChVT:QNG41875.1

FiTA:QED42804.1

TrTA:QED42832.1

GVA:NP 619662.1
ACVB:YP 004935358.1

ASGV:NP-044335.1

YaVA:YP-009268859.1

RAVA:YP $\overline{0} 0053496.1$

ACLSV:NP 040551.1

CtChV-1:YP 009103999

CtChV-2:YP-009103996.

DiVA:YP $00 \overline{6} 905850.1$

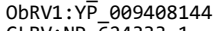

CLBV:NP $\overline{6} 24333.1$

CLBV: AFĀ43536.1

AVCaV:YP 008997790.1

WVA:YP_匂9357235.1

WVA:QEĀ69426.1

CRMaV:YP_007761581.1

CTLaV:YP_009046478.1

GCLV:YP 004936159.1

ASPV:NP 604464.1

APV1:YP_009094347.1
YNVN-RNTRLALAGDDMYAVGKLQ--LRRDREDLLDK-FTLKAKVQFTESPMFCGWYMTPFGIIKEPRLVLERWLIAEEKGTLKECIINYAIEVSYGYRLGEYQYEVIK---NIE 1547 YNIT-RDTVIAFAGDDMYASGKLE--IRKDREDLLAH-LTLKAKVQFTEKPMFCGWYIKKMGIVKEPRLVLERWLIAERKKVIDQCFINYSIEVSYGYRLGEYLWEYFD---NLE 1549 YNIT-RDTVIAFAGDDMYASGKLE --IRKDREDLLAH-LTLKAKVQFTEKPMFCGWYIKKMGIVKEPRLVLERWLIAERKKVIDQCFINYSIEVSYGYRLGEYLWEYFD---NLE 1549 YNIT-RDTVIAFAGDDMYASGKLE --IRKDREDLLAH-LTLKAKVQFTEKPMFCGWYIKKMGIVKEPRLVLERWLIAERKKVIDQCFINYSIEVSYGYRLGEYLWEYFD-- -NLE 1550 YNIS-RDTVIAFAGDDMYASGRLE --IRKDREDLLSH-LTLKAKVQFTDKPMFCGWYIKKKGIVKEPRLVLERWL IAERKKVIDQCFINYSIEVSYGYRLGEYLWEYFD---NLE 1550 YKIN-KNTPIAFAGDDMFSPGRLE--VRRDREFLLNR-FSLKAKVNFSKEPMFCGWRMTPYGIVKEPKLVLERFKIAEERGCFKECLINYCLEVSFAYRLGERLYDVIK---NIQ 1721

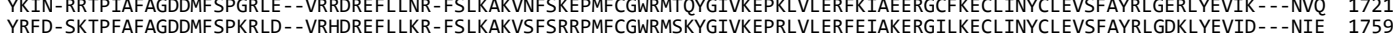
YRFD-SKTPFAFAGDDMFSPKRLD--VRHDREFLLKR-FSLKAKVSFSRRPMFCGWRMSKGGIVEPRLVLERFEIAKERGILKECLINYCLEVSFAYRLGDKLYEVID---NIE 1759
YKFD-SKTPFAFAGDDMFSPRLDD--VRYDREFLLKR-FSLKAKVSFSRKPMFCGWRSKYGIVKEPRLVLERFEIAKERGILKECLINYCLVSFAYRLGDKLYEVID---NIE 1759 YEIN--GLPIAFAGDDMFAPGLLK - - IRKDREFLLDR-FSLKAKVNFSRKPMFCGWRMTRYGIIKEPKLVLERFRIAKERGAFOECLINYCLEVSFAYRLGDRLYDLIE---NIE 1724 YEIN--GLPIAFAGDDMFAPGFLK --IRKDREFLLNR-FSLKAKVNFSRKPMFCGWRMTRYGIIKEPKLVLERFKIARERGTFQECLINYCLEVSFAYRLGDRLYDLIE---NIE 1724 YKID-CKTPICFAGDDMFSPGRLS--LRHDREFLLER-FKLKAKVNFSLNPMFCGWRMTKFGIVKEPKLILERFRIARERGNFRECLINYCLEASFAYRLGDRLYDVIK ---NIE 1740 YHID-RNTPMCFAGDDMYSPGILR --VKKDYEATLDQ-LTLKAKVHISEEPLFCGWRMSPFGIIKEPNLILDRWKIALRSGNLSLCLVNYAIEASFGYRLSEHLYDV---NIDVD 1656

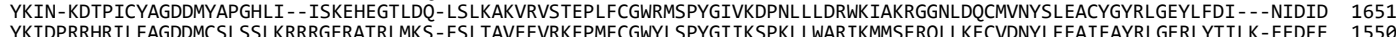
YKIDPHRCRILFAGDDMCSLTRLRKRNSKESQRLLSQ-FSLEAVEESRKFPMFCGWYLSPYGILKSPKLLWARIAMMRERNLLAECVDNYLFEAIFAYRLGERLYRILK-EEDVE 155 YSGA-EGFPILFAGDDMCIFGQIS--ESKGGKELLDRCLRLKSKTFRKYDPEFCGWRLTPLGIFKDPILMYTRTKLHHEQGRLKEVINSYALELAFGYRLGDYCYEFMT -EEQMG 1790 YEVP-DGTPICFAGDDMCALRNLR--EIDTHEFILSK-LSLKAKVNRTKVPMFCGWRLCCDGLIKEPCLIYERLQVAIENGRLMDVIDSYFLEFSFAYKLGERLYSHLE-IEQLN 1822 YQMS-GHEPICFAGDDMCALADLK--ESDEYNAFFKS-FSLKAKVCRTVPPLFCWRLTKFGLYKEPVLVYERLKIAIEKDKLDLVIDSYFLEFCYAYKLGSWLDWVLD-EEQAD 1793 YNV-RTTPICFAGDDMCILTNAK --IRNEMNDFIGS-LKLKAKVEWKINPIFCGWILSRRGILKLPSLVYYRLNIAKEKGNLKDCIDSYMIEAGYAYRKGAFIEELLD-EDQMS 1822 YDLN-GTESICFAGDDCCNRGIKARVDGKYDHLKR-LTKAKAVITKEPTFCGIRLTKYGIFKKPELVLERFLIAIEKGRLDVIDSYYIECSYAYMLERLFECFS - EKDFS 1636 YDLN-GTESICFAGDDMCCNRGIKARVDGKYDHILKR-LTLKAKAVITKEPTFCGWRLTKYGIFKKPELVLERFLIAIEKGRLLDVIDSYYIECSYAYNLGERLFECFS-EKDFS 1636
YDLT-GKEAICFAGDDMCCNKGIRRRTDGRFDHILNR-LSLKAKAVITTEPTFCGWRLTKYGIFKKPELVLERFLIAIEKNKLKDVIDSYYLECSYAYSLGERLFECFS-EKDFV YEWR-RGOPIAFAGDDMCALNNLA--VCHDFDDLFEL-ISLKAKVERTETPMFCGWRLTPYGIVKEPELVYNRFOVAIEEGKVLECLENYAIEVSYAYSLSERLYEVLKSEROVO 1929 YEWR-RGOPIAFAGDDMCALNNLP - ICHDFDDLFEL - ISLKAKVERTESPMFCGWRLTPYGIVKEPELVYNRFOIAIEEGKVMECLENYAIEVSYAYSLSERLYEVLKSEROIO 1954 YEWN-SGDPIAFAGDDMCALKNLK --VTDOFNNVFEK-ISLKAKTOITEVPMFCGWRLSRFGIVKEPELVYNRFMVALERGNVKDCLENYAIEVSYAYSLGERLFDILKREEOLE 1646 YECD-HKTPIAFAGDDMCMLKACK --VSDKFEDVLSK-LSLKAKVIRTEMPMFCGWNLSRYGIVKEPELVFNRFMVAKKRGNIDECLENYAIEVSYAYSLGEKLYEVLKREEQVE 1957 YEWR-RGMPIAFAGDDMCSLSNLK --LSDRFEDLFDK-ISLKAKTQWTEEPMFCGWRLSRHGIVKEPELVFNRFMVALEEGKVELCLENYAIEVSYAYRLGEKLYEVLKSERQIE 1791 YEWR-RGMPIAFAGDDMCSLSNLK --VSDRFEDLFDK-ISLKAKTQWTEEPMFCGWRLSRHGIVKEPELVFNRFMVALEEGKVELCLENYAIEVSYAYRLGEKLYEVLKSERQIE 1791 YEIN-GREAICFAGDDMCANKLLR--KKSEFEHILDR-MTLKAKVQHTTEPTFCGWRLGNFGIVKRPQLVQERILIALEKGNFHECIDNYAIEVSYAYNLGERLISIMS-EKELD 1989 YETN-GRESICFAGDDMCANKLLR--KKKEYEHVLR-MTLKAKMQHTTEPTFCGWRLGPFGIIKRPQLVQERIPIALEKGNFNECIDNYAIEVSHAYNLGDRLISIMS-EKELD 1999 YEIK-GHERTCFAGDDMCANARL YDLN-GREAICFAGDDMCANSRLK--VTNRFSNFDK--IKLKAKVQFTATPTFCGWGLCEHGVFKKPDLVLERDIARETRNLENCIDNYAIEVSCAYKMGENLNLYLT-PEVV 2150 YDLN-GSEAICFAGDDMCANRRLR--VSKKNENFLGK-IKLKAKVQFTEKPTFCGWNLCMDGIFKRPQLVLERLCVAREKDNLANCLDSYAIEVGYAFALGEKILQYMD-EEALQ 1994

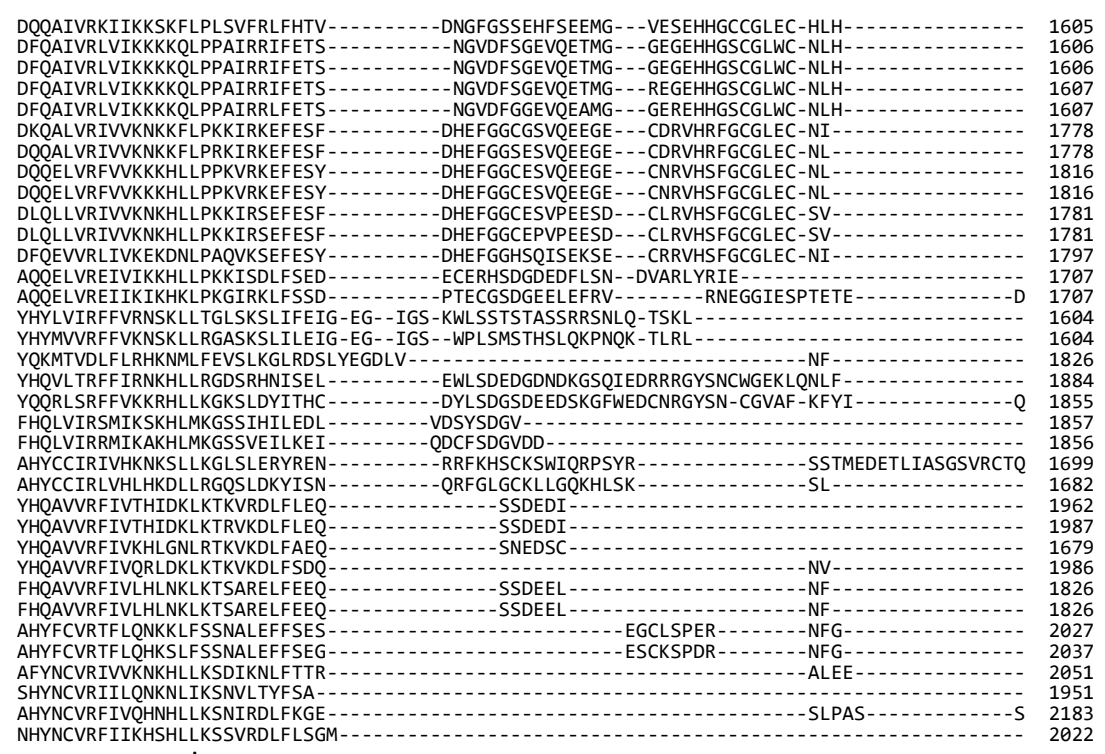

$$
\therefore \text {. }
$$


Fig. S2. Alignment of movement protein sequences of $\mathrm{AgVT}$ and related viruses

AgVT
PVT:YP_002019749.1

PVT : ADX 41472.1

PVT:AFU55322.1
PVT:AXK90540.1

PVT : AXK90540.1
PrVT:YP 009051685.1

PrVT:AHM̄92767.1

FiTA:OED42805.1

FITA:QED42805.

ZoVT: OBS17032.1

TrTA:OED42833.1

GVA:NP 619664.1

ACVB:YP_004935360.1

AgVT
PVT:YP_002019749.1

PVT : ADX 41472.1

PVT:AXK90540.1

PrVT:YP 009051685.1

PrVT: AHM 92767.1
ChVT: ONG41876.1

FiTA:QED42805.

ZoVT:QBS17026.

ZoVT:QBS17032.

TrTA:QED42833.1
GVA:NP 619664.1

GVA:NP 619664.1

GPGV:YP 004732979.2

AgVT
PVT:YP 002019749.1

PVT: AD̄ X14772.1

PVT: AFU55322.1
PVT:AXK90540.1

PrVT:YP 009051685.1

PrVT: AHM92767.

ChVT: QNG41876.1

ZOVT:OBS17026.1

ZoVT:QBS17026.1

TrTA:

GVA:NP 619664.1

AcVB:YP_004935360.1
GPGV:YP_004732979.2

AgVT

PVT:YP_002019749.1

PVT : ADX̄ 41472.1

PVT:AXK90540.1

PrVT:YP 009051685.1

PrVT:AHM̄92767.1

ChVT: QNG41876.1

FiTA:QED42805.1

ZoVT:QBS17026.

ZoVT:QBS17032.

TrTA:QED42833.

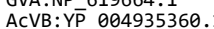

GPGV:YP ${ }^{-} 004732979.2$

AgVT
PVT:YP_002019749.1

PVT : ADX̄ 41472.

PVT:AFU55322.1
PVT:AXK90540.1

PrVT:YP_009051685.

PrVT:AHM92767.1

FiTA:QND42805.1

ZOVT:OBS17026.1

ZOVT:QBS17032.1

TrTA:OED42833.

GVA:NP 619664.1

AcVB:YP_004935360.1

MAMEME- ME--- LISVEKFRRQWEERESITGP---VDSGAIYTNSAFHNLKTKWHVYKSECSIGLDLPD-NGKIISKDIPLFDQEEIDNIIKDDKQVFVHL ME---LISVEKFRRQWEEKESITGP---VDSGAIYTSSPFHNLKTKWHVYKSECSVGLDLPD-NGKIISKDIPLFDQEEIENIMKDDKQVFVHL MSMS - L MS MNMN-1-1 MS - - - - - - VDSSAIYDLSPFHRLSRSTYVRKSEFKVKLEOSG-EKGVASMSIPLFDEIDKAT-IKDSGMPYVHI MS --QEGSLGTKASSFEPQ- - - -DIKVFHVKRSTRDLETLNKS--- - - LHRGDVYNTELIEKVFPR- - -RTKKCVIHKDVIV-KDGRVDCDLDIMDEG - LDD-INEEEFPLYHV MSSISRGSSSMTSSSTVPRAIAKSTKIFDVPKG -EDGRSVARA---- - LNRNRVYKMDAFEKIFHQ- - -STLKSCVHDELIV-ENGVVDQNIDLVDEKTIDG - LNEEKOPYLHL

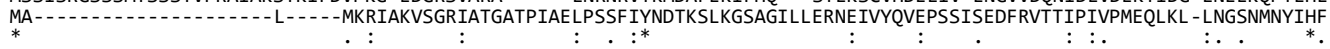

GALIFGIEALF--KVDQDLEVYCFI - -VDRRRTDLESAIIGSSSKLNMGMGRAAFCVRPNFSVAKEDLEDCMSISAIVYARNLRMRAGYRPFALSGGAIYRCTNTSFARSKLKK GAFVFGLVAHF - -PVDEEVEGLVSI - - IDKRRTDLRRATLACRKIKFVNGRCAFMMKPNFSVRKEDLRDGDTFCAAIKIKNLGFEGGFFPFSACGGVIYRTSNVSFAHAVDKT - F 作 GAFVFGLVAHF --PVDEEVEGLVSI - IDKRRTDLRRATLACRKIKFINGRCAFMMKPNFSVRKEDLRDGDTFCAAIKIKNLGFEGGFFPFSACGGVIYRTSNVSFAHAVDKT-F AVVLIOISCLFDWAISEGMEGTFAL --MDTLFDNVQDNVIRACHFRFEEGRAACCFKLNFPICAEDALKGRPIVPYIKVVGANVREGLRGFSVSVGTIFSLNKTEFPSVPMKI AVVLIQISCLFDWSMTNGMEGTFAL - - LDTLFDNVKDNIIRACNFKFVDGRAACCFRMNFSICAEDAMSGRPIVPYIKVLGANIRDGLRGFSVSVGTIYSLNRTEFPSVQLKV - AVVLIQISCLFDWSMTDGMEGTFAL - -LDTLFDNVKDNIIRACNFRFVDGRAACCFRMNFSICAEDAMSGRPIVPYIKVLGANIRDGLRGFSVSVGTIYSLNRTEFPSVOLKV -AAVLIQISCLFDWAISEKMEGIFAL--MDTLFEDPEENVVRACNFRFVDGRAAGCFRMNFSICAEDAIKGRPLIPYIKVKGVNIREGLHGFSVSIGTIFSLNKTEFPSVPLKI AAVLIQISCLFDWAISEKMEGIFAL --MDTLFEDPEENVVRACNFRFVDGRAAGCFRMNFSICAEDAIKGRPLIPYIKVKGVNIREGLHGFSVSIGTIFSLNKTEFPSVPLKI - ACIV GCIVVALM----PHGKNLQGKVSVEVLDTRLVDGA-SRISRTLMDMSKPLSACADFPGYISTSDLLNGYTLHLSITTTDLQFVDGVHPFSVQLMSIGRFCGEDMKTRYAITET GALSISIDPLF --RRDSGVKGVAFV - - YDSRWDNASQALLOAFHFDLNNGTASMVCSPNYSVOLSDPRLSTCLSAVLLFENLNFKSGSYAISVRIGITYRPFNSNIGRSLMS - - . : $:{ }_{*}::_{*}$

SACSVDDLLQSEILELSDLSDESVNSL - - REDVERRVPLLEY--PDEKDY-ASRTVHDLVGTDILSLDQLDRATLEDL - - EE - -VRRSPILRLTAPDERVMASRTVHDLVGTDILSLDQLDQATLEDL - - EE - VRRSPILRLTAPDERVM-

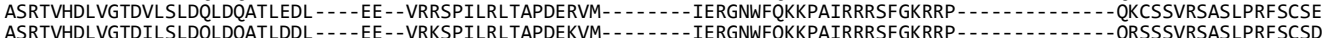
ASRTVHDLVGTDILSLDQLDQATLDDL---EE-VRKSPILRLTAPDEKVM--

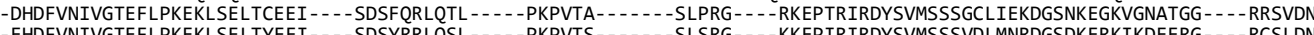
-EHDFVNIVGTEFLPKEKLSELTYEEI---SDSYRRLQSL- -

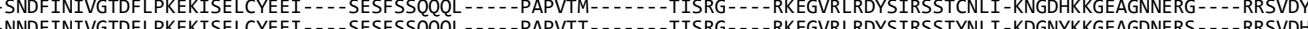

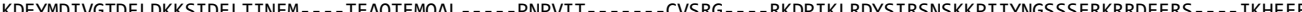
-KDEYMNIVGTDFLDKKSIDELTINEM - TEAQTEMQAL - PNPVVT- 作 KPGAYQELTEG-DGELI--

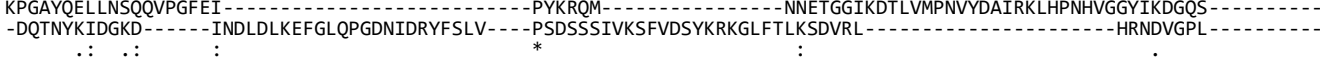

-DLGRRLLSIKDGPSNSQRSEGRSSGDDGHNVLRELQINRGEQGPRGT -GDAVPSR - - - IHIRDDSDQGSERESQVGELGHRVRDFQL - - - -

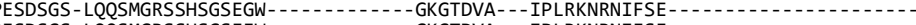
RLERGFES-ESLAGLVLDQKYGSNNFCADKRRSPESDSGSS-LQQSMGQSSHSGSEEWRIERGFES-ESLAGIVLDKKYGPNNFRSDKGGSSESDCGS-LQQPMGRSGHSSPERW- - - -

KVQTSSKTGPAVLRRCSSFSQGDDLWEHSTEGSKRTNRVR-RSGSVKRLVRSGVRGA- - - PRADGPGKGISRGKVOTAGEAGSTILRRSSSISEGNGVREHRSERGKRTDRIR-GSRSVKRLVRAGVRGA- - - - -SWTDGSGGGVSRR-_-

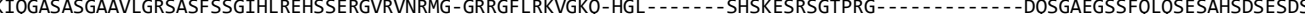
KIQGTSTSGAAVLNRSASFSSGVHFREYSSEGGVRVDRMG -GRRGHLRKVGKQ-HRF-EVWKS-ASRFSLDKRPVLASSSVRIREYCSEGSKSDDGIR-ELCSGERRVFGGRRGTGREFTGTSWADFRGRGTDGGSSSNNNKEDQIEGTNSSSKGSECGVQSELLHSSK - - -EVWKS-ASRF SLDKRPVLASGSVRIREYCSEGSKSDDGIR-ELCSGERRVFGGRGGGREFTGTSWSDFGGRGTNGGSSSNNNKEDKVEDASSSSKGSECGLQSELLHSSK --_-EVQTSDASRHSLRRSNSAIHSGIHLRKHSSEGSFKFNGMG -GHRGSNGPLYSG-SGWG----- SLHKELGGTSSGG \begin{tabular}{l}
379 \\
$-\quad 396$ \\
$-\quad 396$ \\
\hline
\end{tabular} GGSSNIKEDKVEDSSSSKGSECGLQSELLHSSK---S KGDNRRVGVGESPTN 
Fig. S3. Alignment of coat protein sequences of AgVT and related viruses

AgVT
PVT:YP 002019750.1
PVT:AFŪ55323.1
PVT:ADX41473.1

PVT: ADX41473.1
PVT:AXK90541.1

PrVT:AHM92768.1

PrVT:YP 009051686.1

ChVT: QNG 41877.1

FiTA:QED42806.1

TrTA:QED42834.1

ZoVT:QBS17033.1

GVA:NP 619665.1

GPGV:YP- 004732980

AgVT
PVT:YP_002019750.1

PVT:AFŪ55323.

PVT :ADX41473.1

PVT:AXK90541.1

PrVT:AHM92768.1

PrVT:YP 00905168
ChVT: QNG41877.1

FiTA:ONC42806.1

TrTA: QED42834.1

ZoVT:OBS17027.1

ZoVT:QBS17033.1

GVA :NP 619665.1

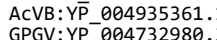

AgVT

PVT:YP_002019750.1
PVT:AFŪ55323.1

PVT : AFU 55323.1
PVT :ADX41473.1

PVT : ADX41473.1
PVT :AXK90541.1

PVT:AXK90541.1
PrVT:AHM92768.1

PrVT:YP 009051686.1

ChVT: QNG 41877.1

FiTA:QED42806.1

TrTA:QED42834.1

ZoVT:QBS17027.1

ZoVT: QBS17033.1
GVA:NP 619665.1

GVA: NP 619665.1

GPGV:YP-004732980.2
M- -DPQTLKDLKAEVLEMTATMYSGSFKSIEGNKDLEELAMQYLLEFIFGMIAIRGASEKVK -WENSGIESETFN - -FEVERKKTTPGAGAASASVTI -

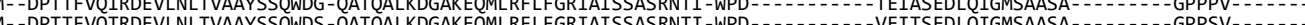
-DPTTFVOIRDEVLNLTVAAYSSOWDG-OATOALKNGAKEQMLRFLFGRIAISSASRNTI-WPD- -

M--DPTTFVOIRGEVLNLTVAAYSSOWDG-OATOALKDGAOEOMLRFLFGRIAISSASRNTL-WPD-

MGLTKKERSKMRKEVDVLLITKFKPQV---KQGQOFSDAAQAFLKEMVFGNIALKGSEQTE-FED-

MGLTKKERSEMRREVDALLITKFKPOV - - KOGOOFSDAAOAFLKEMIFGNIALKGASEOTE-FEDMGITKKERQE IMKEVDGVLTTKFKGQV---PQGQQFSVEVLHFLQEYIFGNIALKGASESTE-WED-MGITKKERLEIMKEVDGVLTTKFKGQV---PQGQQFSIEVLHFLQYIFGNIALKGASELTE-WED--

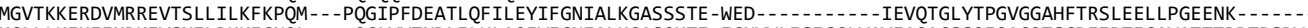
MGLLLKEKEEMRKEVSNILRKKFGNQL ----QGLVWTNDLFLLHLAQFVFGNIALKGASQMTE-FGNYVLESEGCLVAVEAQAGSSQEQAGQTSGDEEPTEGAVATTTRRTRSRVQ MGLLLKEKEEMRKEVSNVLRKKFGNQL---QGLVWTNDLFLHLAQFVFGNIALKGASQMTE-FGNYVLESEGCLVAVEAQAGSSQEQAGQTSGDEEQTEGAAATTTRRTRSRMQ MSGATSRTNLRKEIEELVLTGVVIAPDA- - KTAGYDKGMYLRTLFGYIALNGTSKKTTHYDD - -

M----SIRQELRSTVRRELIAKLSEAN---QVLHGLTEGNKDLVLDHIFANI$$
:^{*}, *^{*}::^{*},
$$

- EKETVGVSFKVNFLTYTQAVRVLLTSSKNKRIKGKTFRQACIPFAEHAKNYLLGHPEC --KTAIFAKAPKSAGRAPHVAFDFAEGLNYN- - - ILKNEEKSVIQWMSARLFKTQ 作

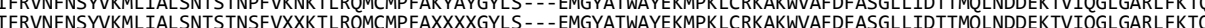

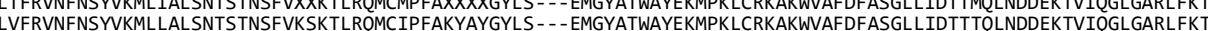
--ALRDGAYRFKVNFFSLVQSLIALLRGSNNVFVNNKPFRRLCVAYASEAKAYLEAKKVDGEFSNLVLKMPATCKHAPEVCFDFNEGLDVL - - -RLTDVOAQTMORLSRRLFATE - ELRAAAFNFKVNLHTLTOSLTALANSSSNVFVSNKSLRRLCVPYAERAKTYLSIRKVDGEYTNLVOKMPDTCRLAPEVCFDFNEGLDVL - - -KLTDVOARVMOKLORRLFATE 作 A IPVPREVNVVFVVNFTLVKSFNMLLSTSENTFVRNKTLRRLCLPFADEAKTYLEIAKGYGEYTTLVTKMPGTCKHAPEVCFDFNEGLDVG - - RLTDVQTSVMOKTORRLFHTE VPAPREVNVVFVVNFFTLVKSFNMLLSTSENTFVRNKTLRRLCLPFADEAKTYLEIAKGYGEYTTIVVTKMPGTCKHAPEVCFDFNEGLDVG---RLTDVOTSVMOKIORRLFHTE ---ETSIDSRGKINVATAVROMLSFSRITPTGAAKGATLROMCEPFAEEARECLAILASKGIYSOLATKLSKLGQKEPQVMFDFNGGLDLG---RMSATEAATTQSLNSRLFRTE - FNPSLPVVKEYSLAEVVNKIRVYKESHTNNDIKLMTFRQVCAAFAIDAQLGLVKFYRLNMHTNIYKKHPKLCDKAPEVAFDFNEGLNFN - - -NLTPNQKSVIQNLNRLLFHVE
..
.

GTIETQNAYIESQDSGREL 220 QSIQIADSTMDGEAINREI QSIQIADSTMGEAINREI IKKRESERASEDH-VGDOV LKKRESERSVADH-IGDOV KKRESDRVSEDH-IGDQL LKKRESDRVSEDH-IGDQL LKKNESDKLTNDH-VGDQV KAKRESEARINEG-VGEEV KAKRESEARINEG-VGE -GAKGVFNAQSSIGEQAVEI SEKOKTNAITASGESAFSM 19 